\title{
Explicit Growth and Expansion for $\mathrm{SL}_{2}$
}

\section{Emmanuel Kowalski}

ETH Zürich - D-MATH, Rämistrasse 101, 8092 Zürich, Switzerland

Correspondence to be sent to: kowalski@math.ethz.ch

We give explicit versions of Helfgott's Growth Theorem for $\mathrm{SL}_{2}$, as well as of the Bourgain-Gamburd argument for the expansion of Cayley graphs modulo primes of subgroups of $\mathrm{SL}_{2}(\mathbf{Z})$ which are Zariski-dense in $\mathrm{SL}_{2}$.

\section{Introduction}

Our main goal in this paper is to prove the following result, which is an explicit version of a theorem of Bourgain and Gamburd [1]:

Theorem 1.1. Let $S \subset \mathrm{SL}_{2}(\mathrm{Z})$ be a finite symmetric set such that the subgroup generated by $S$ is Zariski-dense in $\mathrm{SL}_{2}(\mathbf{Z})$. Let $\mathcal{P}$ be the set of primes such that $S_{p}=S(\bmod p)$ generates $\mathrm{SL}_{2}\left(\mathbf{F}_{p}\right)$, which contains all but finitely many primes. Then the family of Cayley graphs $\left(\mathcal{C}\left(\mathrm{SL}_{2}\left(\mathbf{F}_{p}\right), S_{p}\right)\right)_{p \in \mathcal{P}}$ is an expander family, and one can write down explicit bounds for the spectral gap, given the set $S$.

In particular, if $S$ generates a free group of rank $|S| / 2$, the spectral gap (This is the spectral gap of the normalized Laplace operator $\Delta=\mathrm{Id}-M$, where $M$ is the Markov averaging operator of the graph; thus the spectrum of $\Delta$ is a subset of the interval [0,2].) satisfies

$$
\lambda_{1}\left(\mathcal{C}\left(\mathrm{SL}_{2}\left(\mathbf{F}_{p}\right), S_{p}\right)\right) \geq 2^{-2^{35} \gamma^{-1}}
$$


for all $p$ large enough, where

$$
\gamma=\frac{\log \left(\frac{2}{\sqrt{3}} \sqrt{|S|}\right)}{\log \max _{s \in S}\|S\|},
$$

the norm $\|s\|$ being the operator norm of the matrix $s$, with respect to the euclidean metric on $\mathrm{C}^{2}$.

We can specify what " $p$ large enough" means, but we defer a statement to Section 4.3 since this involves a series of inequalities which are awkward to state (and unenlightening), but easy to check for a given concrete set of matrices $S$.

A crucial ingredient in the argument of Bourgain and Gamburd is Helfgott's Growth Theorem [11] for $\mathrm{SL}_{2}$, which has considerable independent interest. We thus require an explicit version of it, and we will prove the following:

Theorem 1.2. Let $p$ be a prime number, $H \subset \mathrm{SL}_{2}\left(\mathbf{F}_{p}\right)$ a symmetric generating subset of $\mathrm{SL}_{2}\left(\mathbf{F}_{p}\right)$ containing 1 . Then the triple product set $H^{(3)}=H \cdot H \cdot H$ satisfies either $H^{(3)}=$ $\mathrm{SL}_{2}\left(\mathbf{F}_{p}\right)$ or

$$
\left|H^{(3)}\right| \geq|H|^{1+\delta},
$$

where $\delta=\frac{1}{3024}$.

Here is a simple corollary, which is (as far as the author is aware) also the first explicit result of this kind for almost simple linear groups:

Corollary 1.3 (Explicit solution to Babai's conjecture for $\mathrm{SL}_{2}\left(\mathrm{~F}_{p}\right)$ ). For any prime number $p$ and any symmetric generating set $S$ of $\mathrm{SL}_{2}\left(\mathbf{F}_{p}\right)$, we have

$$
\operatorname{diam} \mathcal{C}\left(\mathrm{SL}_{2}\left(\mathbf{F}_{p}\right), S\right) \leq 3\left(\log \left|\mathrm{SL}_{2}\left(\mathbf{F}_{p}\right)\right|\right)^{C}
$$

with $C=3323$.

Another corollary of Helfgott's Theorem and of intermediate results used in the proof of Theorem 1.1 is a better diameter bound for Zariski-dense subgroups:

Corollary 1.4 (Diameter bounds for Zariski-dense subgroups of $\left.\mathrm{SL}_{2}\right)$. Let $S \subset \mathrm{SL}_{2}(\mathrm{Z})$ be a finite symmetric set such that the subgroup generated by $S$ is Zariski-dense in $\mathrm{SL}_{2}(\mathbf{Z})$ and is a free group of rank $|S| / 2$. Let $\mathcal{P}$ be the set of primes such that $S_{p}=S(\bmod p)$ generates $\mathrm{SL}_{2}\left(\mathbf{F}_{p}\right)$. 
Let $\delta>0$ be as in Helfgott's Theorem and define

$$
\tau^{-1}=\log \max _{s \in S}\|s\|>0
$$

Then for $p \in \mathcal{P}$ and $p>\exp (2 / \tau)$, we have

$$
\operatorname{diam}\left(\mathcal{C}\left(\mathrm{SL}_{2}\left(\mathbf{F}_{p}\right), S\right)\right) \leq 3^{A}\left(\log \left|\mathrm{SL}_{2}\left(\mathbf{F}_{p}\right)\right|\right)
$$

where

$$
A=\frac{\log \left(8 \tau^{-1}(|S|-1)^{-1}\right)}{\log (1+\delta)} .
$$

Remark 1.5. Using the well-known bound

$$
\lambda_{1}\left(\Gamma_{p}\right) \geq \frac{1}{|S| \operatorname{diam}\left(\Gamma_{p}\right)^{2}}
$$

(see, e.g., [18, Theorem 13.23]), these diameter bounds can be used to obtain lower bounds for spectral gaps for "medium" primes. Note the huge discrepancy however at the end of the range.

Combining Theorem 1.1 with the second corollary, we can give explicit statements for the motivating example of the Lubotzky group.

Corollary 1.6 (The Lubotzky group). Let

$$
S=\left\{\left(\begin{array}{cc}
1 & \pm 3 \\
0 & 1
\end{array}\right),\left(\begin{array}{cc}
1 & 0 \\
\pm 3 & 1
\end{array}\right)\right\} \subset \mathrm{SL}_{2}(\mathrm{Z})
$$

and let $\Gamma_{p}=\mathcal{C}\left(\mathrm{SL}_{2}\left(\mathbf{F}_{p}\right), S_{p}\right)$. Then we have

$$
\lambda_{1}\left(\Gamma_{p}\right) \geq 2^{-2^{36}}
$$

if $p \geq 2^{2^{46}}$, and

$$
\operatorname{diam}\left(\Gamma_{p}\right) \leq 2^{5572}\left(\log \left|\mathrm{SL}_{2}\left(\mathbf{F}_{p}\right)\right|\right)
$$

for all $p \neq 3$. 
The original papers of Bourgain and Gamburd [1] and Helfgott [11] are effective, and thus it is not surprising that one can obtain explicit versions. What is less clear is how good the constants may be, and how much work may be required to provide them. This paper gives a first indication in that respect.

The bounds we derive are very unlikely to be anywhere near sharp, and not only because we often use rather rough estimates to simplify the shape and constants appearing in various inequalities. (In some cases, one can easily extract better bounds from the proof, e.g., one can replace $\frac{1}{3024}$ by $\frac{1}{1513}$ for all $H$ large enough in Theorem 1.2.) Indeed, when the Hausdorff dimension of the limit set of the subgroup $G$ generated by $S$ is large enough, Gamburd [8] has shown quite good spectral gaps for the hyperbolic Laplace operator on $G \backslash \mathbf{H}$, which strongly suggest that the corresponding combinatorial spectral gap would also be relatively large. But this computation has not been done, to the author's knowledge, and our version of Theorem 1.1 gives the first fully explicit spectral gap for infinite-index subgroups of $\mathrm{SL}_{2}(\mathbf{Z})$, with Corollary 1.6 being a nice concrete example (it is also known that the "Lubotzky group" is too small for Gamburd's result to apply).

In view of the direct link between the spectral gap of families of Cayley graphs of quotients of "thin" (or sparse) subgroups of arithmetic groups and quantitative applications of sieve methods to these groups, it is natural to wish for a better understanding of these issues. (Indeed, this question was asked by J-P. Serre during the author's Bourbaki lecture [15].) A first step towards effective versions of these applications of "sieve in orbit" would be to extend Theorem 1.1 to an effective spectral gap for $\mathrm{SL}_{2}(\mathrm{Z} / q \mathrm{Z})$, where $q$ is a squarefree modulus (as originally proved by Bourgain, Gamburd, Sarnak [2]), and we hope to come back to this.

As a final remark, the reader can also see this paper as presenting a complete proof of the qualitative forms of Theorems 1.1 and 1.2 and their corollaries. When read in this light, ignoring the fussy technical details arising from trying to have explicit bounds, it may in fact be useful as a self-contained introduction to this area of research.

Notation. As usual, $|X|$ denotes the cardinality of a set. Given a group $G$, and a symmetric generating set $S$, we denote by $\mathcal{C}(G, S)$ the Cayley graph of $G$ with respect to $S$, which is $|S|$-regular. Moreover, we say that a symmetric set $S \subset G$ freely generates $G$ if representatives of $S$ modulo the relation $s \sim s^{-1}$ form a free generating set of $G$, that is, $G$ is a free group of $\operatorname{rank}|S| / 2$.

For a subset $H \subset G$ of a group $G$, we write $H^{(n)}$ for the $n$-fold product set

$$
H^{(n)}=\left\{x \in G \mid x=h_{1} \cdots h_{n} \text { for some } h_{i} \in H\right\} .
$$


Note the immediate relations

$$
\left(H^{(n)}\right)^{(m)}=H^{(n m)}, \quad H^{(n+m)}=H^{(n)} \cdot H^{(m)}
$$

for $n, m \geq 0$ and $\left(H^{(n)}\right)^{-1}=H^{(n)}$ if $H$ is symmetric. In addition, if $1 \in H$, we have $H^{(n)} \subset$ $H^{(m)}$ for all $m \geq n$. In particular, the diameter of a Cayley graph $\mathcal{C}(G, H)$, when $H=H^{-1}$, is the smallest $n \geq 1$ such that $\tilde{H}^{(n)}=G$, where $\tilde{H}=H \cup\{1\}$.

We denote by $\operatorname{trp}(H)$ the "tripling constant" of a subset $H \subset G$, defined by

$$
\operatorname{trp}(H)=\frac{\left|H^{(3)}\right|}{|H|} .
$$

\section{Explicit multiplicative combinatorics}

Another ingredient of Theorem 1.1 is the relation between subsets of a finite group with small "multiplicative energy" and sets with small tripling constant, or approximate subgroups. This was obtained by Tao [24], in good qualitative form, but without explicit dependency of the various quantities involved. In this section, we state a suitably explicit version.

We recall first the definitions involved. For a finite group $G$ and $A, B \subset G$, one defines the multiplicative energy by

$$
E(A, B)=\left|\left\{\left(a_{1}, a_{2}, b_{1}, b_{2}\right) \in A^{2} \times B^{2} \mid a_{1} b_{1}=a_{2} b_{2}\right\}\right| .
$$

It is also convenient to denote by

$$
e(A, B)=\frac{|E(A, B)|}{(|A||B|)^{3 / 2}}
$$

the normalized multiplicative energy, which is $\leq 1$. Following Tao (see [24, Definition 3.8]), for a finite group $G$ and any $\alpha \geq 1$, a subset $H \subset G$ is an $\alpha$-approximate subgroup if $1 \in \mathrm{H}, \mathrm{H}=\mathrm{H}^{-1}$ and there exists a symmetric subset $X \subset \mathrm{H}^{(2)}$ of order at most $\alpha$ such that

$$
\mathrm{H} \cdot \mathrm{H} \subset X \cdot \mathrm{H},
$$

which implies also $\mathrm{H} \cdot \mathrm{H} \subset \mathrm{H} \cdot X$. Then we have:

Theorem 2.1. Let $G$ be a finite group and $\alpha \geq 1$. If $A$ and $B$ are subsets of $G$ such that $e(A, B) \geq \alpha^{-1}$, there exist constants $\beta_{1}, \beta_{2}, \beta_{3}, \beta_{4} \geq 1$, a $\beta_{1}$-approximate subgroup $\mathrm{H} \subset G$ 
and elements $x, y \in G$ such that

$$
\begin{aligned}
& |\mathrm{H}| \leq \beta_{2}|A| \leq \beta_{2} \alpha^{2}|B|, \\
& |A \cap x \mathrm{H}| \geq \frac{1}{\beta_{3}}|A|, \quad|B \cap \mathrm{H}| \geq \frac{1}{\beta_{3}}|B|, \\
& \operatorname{trp}(\mathrm{H}) \leq \beta_{4},
\end{aligned}
$$

and moreover $\beta_{i} \leq c_{1} \alpha^{c_{2}}$ for some absolute constants $c_{1}, c_{2}>0$. In fact, one can take

$$
\beta_{1} \leq 2^{1861} \alpha^{720}, \quad \beta_{2} \leq 2^{325} \alpha^{126}, \quad \beta_{3} \leq 2^{2516} \alpha^{973}, \quad \beta_{4} \leq 2^{930} \alpha^{360}
$$

Except for the values of the constants, this is proved in [24, Theorem 5.4, (i) implies (iv)] and quoted in [26, Theorem 2.48]. Since this is obtained by following line by line the arguments of Tao, we defer a proof to the Appendix.

\section{Growth for $\mathrm{SL}_{2}$}

We prove here Theorem 1.2. The argument we use is basically the one sketched by Pyber and Szabó in [21, Section 1.1] (which is expanded in their paper to cover much more general situations). It is closely related to the one of Breuillard, Greenand, Tao [4], and many ingredients are already visible in Helfgott's original argument [11].

\subsection{Elementary facts and definitions}

We begin with an important observation, which applies to all finite groups, and goes back to Ruzsa: to prove that the tripling constant of a generating set $H$ is at least a small power of $|H|$, it is enough to prove that the growth ratio after an arbitrary (but fixed) number of products is of such order of magnitude.

Proposition 3.1 (Ruzsa). Let $G$ be a finite group, and let $H \subset G$ be a symmetric nonempty subset.

(1) Denoting $\alpha_{n}=\left|H^{(n)}\right| /|H|$, we have

$$
\alpha_{n} \leq \alpha_{3}^{n-2}=\operatorname{trp}(H)^{n-2}
$$

for all $n \geq 3$. 
(2) We have $\operatorname{trp}\left(H^{(2)}\right) \leq \operatorname{trp}(H)^{4}$ and for $k \geq 3$, we have

$$
\operatorname{trp}\left(H^{(k)}\right) \leq \operatorname{trp}(H)^{3 k-3}
$$

Proof. The first part is well known (see, e.g., [11, Proof of Lemma 2.2]). For (2), we have

$$
\operatorname{trp}\left(H^{(k)}\right)=\frac{\alpha_{3 k}}{\alpha_{k}}
$$

Since $\alpha_{k} \geq \alpha_{3}$ for $k \geq 3$, we obtain $\operatorname{trp}\left(H^{(k)}\right) \leq \alpha_{3}^{3 k-3}$ for $k \geq 3$ by (1), while for $k \geq 2$, we simply use $\alpha_{2} \geq 1$ to obtain $\operatorname{trp}\left(H^{(2)}\right) \leq \alpha_{3}^{4}$.

We first use Ruzsa's Lemma to show that Helfgott's Theorem holds when $|H|$ is small, in the following sense:

Lemma 3.2. Let $G$ be a finite group and let $H$ be a symmetric generating set of $G$ containing 1 . If $H^{(3)} \neq G$, we have $\left|H^{(3)}\right| \geq 2^{1 / 2}|H|$.

Proof. If the triple product set is not all of $G$, it follows that $H^{(3)} \neq H^{(2)}$. We fix some $x \in H^{(3)}-H^{(2)}$, and consider the injective map

$$
i:\left\{\begin{array}{l}
H \rightarrow G \\
h \mapsto h x .
\end{array}\right.
$$

The image of this map is contained in $H^{(4)}$ and it is disjoint with $H$ since $x \notin H^{(2)}$. Hence $H^{(4)}$, which contains $H$ and the image of $i$, satisfies $\left|H^{(4)}\right| \geq 2|H|$. Hence, by Ruzsa's Lemma, we obtain

$$
\operatorname{trp}(H) \geq\left(\frac{\left|H^{(4)}\right|}{|H|}\right)^{1 / 2} \geq 2^{1 / 2}
$$

Remark 3.3. In fact, as the referee pointed out, a better result is known (and is elementary): if $H \subset G$ generates $G$, then $\left|H^{(2)}\right| \geq \frac{3}{2}|H|$ (see [25]).

The following version of the orbit-stabilizer theorem will be used to reduce the proof of lower-bounds on the size a set to an upper-bound for another.

Proposition 3.4 (Helfgott). Let $G$ be a finite group acting on a nonempty finite set $X$. Fix some $x \in X$ and let $K \subset G$ be the stabilizer of $x$ in $G$. For any nonempty symmetric subset 
$H \subset G$, we have

$$
\left|K \cap H^{(2)}\right| \geq \frac{|H|}{|H \cdot X|},
$$

where $H \cdot x=\{h \cdot x \mid h \in H\}$.

(Note that since $H$ is symmetric, we have $1 \in K \cap H^{(2)}$.)

Proof. As in the classical proof of the orbit-stabilizer theorem, we consider the orbit map, but restricted to $H$

$$
\phi:\left\{\begin{array}{c}
H \rightarrow X, \\
h \mapsto h \cdot X .
\end{array}\right.
$$

Using the fibers of this map to count the number of elements in $H$, we obtain

$$
|H|=\sum_{y \in \phi(H)}\left|\phi^{-1}(y)\right|
$$

But the image of $\phi$ is $\phi(H)=H \cdot x$, and we have

$$
\left|\phi^{-1}(y)\right| \leq\left|K \cap H^{(2)}\right|
$$

for all $y$ (indeed, if $y=\phi\left(h_{0}\right)$ with $h_{0} \in H$, then all elements $h \in H$ with $\phi(h)=y$ satisfy $\left.h_{0}^{-1} h \in K \cap H^{(2)}\right)$. Therefore, we obtain

$$
|H| \leq|H \cdot X|\left|K \cap H^{(2)}\right|
$$

as claimed.

Finally, a last lemma shows that if a subset $H$ has a small tripling constant "in a subgroup", then $H$ itself has small tripling (in the language of approximate groups, it is a special case of the fact that the intersection of two approximate groups is still one).

Lemma 3.5. Let $G$ be a finite group, $K \subset G$ a subgroup, and $H \subset G$ an arbitrary symmetric subset. For any $n \geq 1$, we have

$$
\frac{\left|H^{(n+1)}\right|}{|H|} \geq \frac{\left|H^{(n)} \cap K\right|}{\left|H^{(2)} \cap K\right|} .
$$

Proof. Let $X \subset G / K$ be the set of cosets of $K$ intersecting $H$ :

$$
X=\{x K \in G / K \mid x K \cap H \neq \varnothing\} .
$$


We can estimate the size of this set from below by splitting $H$ into its intersections with cosets of $K$ : we have

$$
|H|=\sum_{x K \in X}|H \cap x K|
$$

But for any $x K \in X$, fixing some $g_{0} \in x K \cap H$, we have $g^{-1} g_{0} \in K \cap H^{(2)}$ if $g \in X$ $K \cap H$, and hence

$$
|x K \cap H| \leq\left|K \cap H^{(2)}\right|
$$

This gives the lower bound

$$
|X| \geq \frac{|H|}{\left|K \cap H^{(2)}\right|} .
$$

Now take once more some $x K \in X$, and fix an element $x k=h \in x K \cap H$. Then all the elements $x k g$ are distinct for $g \in K$, and they are in $x K \cap H^{(n+1)}$ if $g \in K \cap H^{(n)}$, so that

$$
\left|x K \cap H^{(n+1)}\right| \geq\left|K \cap H^{(n)}\right|
$$

for any $x K \in X$, and (cosets being disjoint)

$$
\left|H^{(n+1)}\right| \geq|X|\left|K \cap H^{(n)}\right|,
$$

which gives the result when combined with the lower bound for $|X|$.

We will use classical structural definitions and facts about finite groups of Lie type. In particular, a regular semisimple element $g \in \mathrm{G}=\mathrm{SL}_{2}\left(\overline{\mathbf{F}}_{p}\right)$ is a semisimple element with distinct eigenvalues. The centralizer of such an element is a maximal torus in G. For any subset $H \subset \mathbf{G}$, we write $H_{\text {reg }}$ for the set of the regular semisimple elements in $H$. A maximal torus $T \subset G=\mathrm{SL}_{2}\left(\mathbf{F}_{p}\right)$ is the intersection $G \cap \mathbf{T}$, where $\mathbf{T}$ is a maximal torus of $\mathbf{G}$ which is stable under the Frobenius automorphism $\sigma$. Here are the basic properties of regular semisimple elements and their centralizers; these are all standard facts, and we omit the proofs. (For general facts about finite groups of Lie type, one may look at [6] or [5, Chapters 1 and 3], and for conjugacy classes in $\mathrm{SL}_{2}\left(\mathbf{F}_{p}\right)$, one may look for instance at [7, p. 70]; another source for $\mathrm{SL}_{2}$ is [23, Chapter 6]). 
Proposition 3.6. Fix a prime number $p$ and let $G=\mathrm{SL}_{2}\left(\mathbf{F}_{p}\right), \mathbf{G}=\mathrm{SL}_{2}\left(\overline{\mathbf{F}}_{p}\right)$.

(1) A regular semisimple element $x \in \mathrm{G}$ is contained in a unique maximal torus $\mathbf{T}$, namely its centralizer $\mathbf{T}=C_{\mathrm{G}}(x)$. In particular, if $\mathbf{T}_{1} \neq \mathbf{T}_{2}$ are two maximal tori, we have

$$
\mathbf{T}_{1, \text { reg }} \cap \mathbf{T}_{2, \text { reg }}=\emptyset \text {. }
$$

(2) If $\mathbf{T} \subset \mathrm{G}$ is a maximal torus, we have

$$
\left|\mathbf{T}_{\text {nreg }}\right|=\left|\mathbf{T}-\mathbf{T}_{\text {reg }}\right|=2 .
$$

(3) For any maximal torus $\mathrm{T}$, the normalizer $N_{\mathrm{G}}(\mathrm{T})$ contains $\mathrm{T}$ as a subgroup of index 2. Similarly, for any maximal torus $T \subset G, N_{G}(T)$ contains $T$ as a subgroup of index 2, and, in particular,

$$
2(p-1) \leq\left|N_{G}(T)\right| \leq 2(p+1)
$$

(4) The conjugacy class $\mathbf{C l}(g)$ of a regular semisimple element $g \in \mathrm{G}$ is the set of all $x \in \mathrm{G}$ such that $\operatorname{Tr}(x)=\operatorname{Tr}(g)$. The set of elements in $\mathrm{G}$ which are not regular semisimple is the set of all $x \in \mathrm{G}$ such that $\operatorname{Tr}(x)^{2}=4$.

Finally, (a variant of) the following concept was introduced under different names and guises by Helfgott, Pyber-Szabó, and Breuillard-Green-Tao. We chose the name from the last team.

Definition 3.7 (A set involved with a torus). Let $p$ be a prime number, $H \subset \mathrm{SL}_{2}\left(\mathbf{F}_{p}\right)$ a finite set and $\mathbf{T} \subset \mathrm{SL}_{2}\left(\overline{\mathbf{F}}_{p}\right)$ a maximal torus. Then $H$ is involved with $\mathbf{T}$, or $\mathbf{T}$ with $H$, if and only if $\mathbf{T}$ is $\sigma$-invariant and $H$ contains a regular semisimple element of $\mathbf{T}$ with nonzero trace, that is, $H \cap \mathbf{T}_{\text {sreg }} \neq \emptyset$ where the superscript "sreg" restricts to regular semisimple elements with nonzero trace.

Remark 3.8. The twist in this definition, compared with the one in [21] or [4], is that we insist on having nonzero trace. This will be helpful later on, as it will eliminate a whole subcase in the key estimate (the proof of Proposition 3.12), and lead to a shorter proof, with better explicit constants. However, this restriction is not really essential in the greater scheme of things, and it would probably not be a good idea to do something similar for more general groups. 
The alternative $H^{(3)}=\mathrm{SL}_{2}\left(\mathbf{F}_{p}\right)$ in Helfgott's growth theorem will be obtained as a corollary of the Gowers-Nikolov-Pyber "quasi-random groups" argument (see [10, 19]).

Proposition 3.9. For a prime $p \geq 3$, if a subset $H \subset \mathrm{SL}_{2}\left(\mathbf{F}_{p}\right)$ satisfies

$$
|H| \geq 2\left|\mathrm{SL}_{2}\left(\mathbf{F}_{p}\right)\right|^{8 / 9},
$$

we have $H^{(3)}=\mathrm{SL}_{2}\left(\mathbf{F}_{p}\right)$.

For a proof, see, for example, [16, Section 4.5].

\subsection{Escape from subvarieties and nonconcentration lemmas}

Two important tools in the proof of growth theorems for linear groups are estimates for escape from subvarieties and for nonconcentration in subvarieties. We state and prove in this section the special cases which we need for the explicit proof of Helfgott's Theorem. The reader may wish to look only at the statements and skip afterwards to the next section to see how they are used.

Lemma 3.10 (Escape). Let $p \geq 7$ be a prime number and let $H \subset \mathrm{SL}_{2}\left(\mathbf{F}_{p}\right)$ be a symmetric generating set with $1 \in H$. Then $H_{\text {sreg }}^{(3)} \neq \emptyset$, that is, the 3 -fold product set $H^{(3)}$ contains a regular semisimple element $x$ with nonzero trace. In particular, there exists a torus $\mathrm{T}=\mathrm{C}_{\mathrm{G}}(x)$ involved with $H^{(3)}$. (The condition $p \geq 7$ is sharp, see [16, Example 4.6.13] for an example).

The general nonconcentration inequalities are now often called "Larsen-Pink inequalities", since the first versions appeared in the work of Larsen and Pink [17] on finite subgroups of linear groups. "Approximate" versions occur in the work of Hrushovski [12] and Breuillard-Green-Tao [4], with closely related results found in that of Pyber and Szabó [21].

Theorem 3.11 (Nonconcentration inequality). Let $p \geq 3$ be a prime number and let $g \in$ $\mathrm{SL}_{2}\left(\mathbf{F}_{p}\right)=G$ be a regular semisimple element with nonzero trace. Let $\mathrm{Cl}(g) \subset \mathrm{SL}_{2}\left(\overline{\mathbf{F}}_{p}\right)=\mathrm{G}$ be the conjugacy class of $g$. If $H \subset G$ is a symmetric generating set containing 1 , we have

$$
|\mathbf{C l}(g) \cap H| \leq 7 \alpha^{2 / 3}|H|^{2 / 3},
$$


where $\alpha=\operatorname{trp}(H)$ is the tripling constant of $H$, unless

$$
\alpha>|H|^{1 / 28} .
$$

From this last fact, we will deduce the following dichotomy, which is the precise tool used in the next section to prove Helfgott's Theorem.

Proposition 3.12 (Involving dichotomy). (1) For all prime number $p$, all subsets $H \subset$ $\mathrm{SL}_{2}\left(\mathbf{F}_{p}\right)$ and all maximal tori $\mathbf{T} \subset \mathrm{SL}_{2}\left(\overline{\mathbf{F}}_{p}\right)$, if $\mathbf{T}$ and $H$ are not involved, we have

$$
|H \cap \mathbf{T}| \leq 4 .
$$

(2) If $p \geq 3$ and $H \subset \mathrm{SL}_{2}\left(\mathbf{F}_{p}\right)=G$ is a symmetric generating set containing 1, we have

$$
\left|\mathbf{T}_{\text {reg }} \cap H^{(2)}\right| \geq 14^{-1} \alpha^{-4}|H|^{1 / 3}
$$

for any maximal torus $\mathbf{T} \subset \mathrm{SL}_{2}\left(\overline{\mathbf{F}}_{p}\right)$ which is involved with $H$, where $\alpha=\operatorname{trp}(H)$, unless

$$
\alpha \geq|H|^{1 / 168} .
$$

Proof. (1) is obvious, since $\left|\mathbf{T}-\mathbf{T}_{\text {reg }}\right| \leq 2$ and there are also at most two elements of trace 0 in $\mathbf{T}$ (as one can check quickly).

For (2), we apply the orbit-stabilizer theorem. Let $T=\mathbf{T} \cap \mathbf{G}$ be a maximal torus in $G$. Fixing any $g \in T_{\text {reg, }}$, we have $T=C_{G}(g)$, the stabilizer of $g$ in $G$ for its conjugacy action on itself. We find that

$$
\left|\mathbf{T} \cap H^{(2)}\right| \geq \frac{|H|}{\left|\left\{h g h^{-1} \mid h \in H\right\}\right|}
$$

for any symmetric subset $H$. Since $H$ is involved with $\mathbf{T}$, we can select $g$ in $T_{\text {sreg }} \cap H$ in this inequality, and the denominator on the right-hand side becomes

$$
\left|\left\{h g h^{-1} \mid h \in H\right\}\right| \leq\left|H^{(3)} \cap \operatorname{Cl}(g)\right| \leq\left|H^{(3)} \cap \mathbf{C l}(g)\right|,
$$

where $\mathrm{Cl}(g)$ is the conjugacy class of $g$ in $G$. Applying the Larsen-Pink inequality to $H^{(3)}$, with tripling constant bounded by $\alpha^{6}$ (by Ruzsa's Lemma), we obtain the lower bound

$$
\left|\mathbf{T} \cap H^{(2)}\right| \geq \frac{|H|}{\left|H^{(3)} \cap \mathbf{C l}(g)\right|} \geq 7^{-1} \alpha^{-4}|H|^{1 / 3},
$$


unless $\alpha=\operatorname{trp}(H) \geq|H|^{1 / 168}$. In the first case, we obtain

$$
\left|\mathbf{T}_{\text {reg }} \cap H^{(2)}\right| \geq 14^{-1} \alpha^{-4}|H|^{1 / 3},
$$

unless

$$
7^{-1} \alpha^{-4}|H|^{1 / 3} \leq 2
$$

since there are only two elements of $\mathbf{T} \cap H^{(2)}$ which are not regular. This last alternative gives

$$
\alpha \geq \frac{1}{2}|H|^{1 / 12}
$$

which we see is a stronger conclusion than (3.6) (precisely, it is strictly stronger if $|H|>$ $2^{13}$, but in the other case the lower bound $\operatorname{trp}(H) \geq \sqrt{2}$ from Lemma 3.2 is already a better result.) Hence Proposition 3.12 is proved.

Now we prove the escape and nonconcentration results.

Proof of Lemma 3.10. The basic point that allows us to give a quick proof is that the set $N \subset \mathrm{SL}_{2}\left(\mathbf{F}_{p}\right)$ of elements which are not regular semisimple is invariant under $\mathrm{SL}_{2}\left(\mathbf{F}_{p}\right)$ conjugation, and is the set of all matrices with trace equal to 2 or -2 . It is precisely the union of the two central elements \pm 1 and the four conjugacy classes of

$$
u=\left(\begin{array}{ll}
1 & 1 \\
0 & 1
\end{array}\right), \quad v=\left(\begin{array}{cc}
-1 & 1 \\
0 & -1
\end{array}\right), \quad u^{\prime}=\left(\begin{array}{ll}
1 & \varepsilon \\
0 & 1
\end{array}\right), \quad v^{\prime}=\left(\begin{array}{cc}
-1 & \varepsilon \\
0 & -1
\end{array}\right)
$$

(where $\varepsilon \in \mathbf{F}_{p}^{\times}$is a fixed nonsquare) while elements of trace 0 are the conjugates of

$$
g_{0}=\left(\begin{array}{cc}
0 & 1 \\
-1 & 0
\end{array}\right)
$$

(these are standard facts, which can be checked on the list of conjugacy classes in [7, p. 70], for instance.)

We next note that, if the statement of the lemma fails for a given $H$, it also fails for every conjugate of $H$, and that this allows us to normalize at least one element to a specific representative of its conjugacy class. It is convenient to argue by contradiction, though this is somewhat cosmetic. So we assume that $H_{\text {nreg }}^{(3)}$ is empty and $p \geq 7$, and will derive a contradiction. 
We distinguish two cases. In the first case, we assume that $H$ contains one element of trace \pm 2 which is not \pm 1 . The observation above shows that we can assume that one of $u, v, u^{\prime}, v^{\prime}$ is in $H$. We deal first with the case $u \in H$.

Since $H$ is a symmetric generating set, it must contain some element

$$
g=\left(\begin{array}{ll}
a & b \\
c & d
\end{array}\right)
$$

with $c \neq 0$, since otherwise, all elements of $H$ would be upper-triangular, and $H$ would not generate $\mathrm{SL}_{2}\left(\mathbf{F}_{p}\right)$. Then $H^{(3)}$ contains $u g, u^{2} g, u^{-1} g$, and $u^{-2} g$, which have traces, respectively, equal to $\operatorname{Tr}(g)+c, \operatorname{Tr}(g)+2 c, \operatorname{Tr}(g)-c, \operatorname{Tr}(g)-2 c$. Since $c \neq 0$, and $p$ is not 2 or 3 , we see that these traces are distinct, and since there are four of them, one at least is not in $\{-2,0,2\}$, which contradicts our assumption.

If $v \in H$, the argument is almost identical. If $u^{\prime}$ (or similarly $v^{\prime}$ ) is in $H$, the set of traces of $\left(u^{\prime}\right)^{j} g$ for $j \in\{-2,-1,0,1,2\}$ is

$$
\{\operatorname{Tr}(g)+2 c,-\operatorname{Tr}(g)-c, \operatorname{Tr}(g),-\operatorname{Tr}(g)+c, \operatorname{Tr}(g)-2 c\}
$$

and one can check that for $p \geq 5$, one of these is not $0,-2$ or 2 , although some could coincide (for instance, if $\operatorname{Tr}(g)=2$, the other traces are $\{2+2 c,-2-$ $c,-2+c, 2-2 c\}$, and if $c-2=2$, we obtain traces $\{2,-6,10\}$, but $-6 \notin\{0,2,-2\}$ for $p \geq 5$ ).

In the second case, all elements of $H$ except \pm 1 have trace 0 . We split in two subcases, but depending on properties of $\mathbf{F}_{p}$.

The first one is when -1 is not a square in $\mathbf{F}_{p}$. Conjugating again, we can assume that $g_{0} \in H$. Because $H$ generates $\mathrm{SL}_{2}\left(\mathbf{F}_{p}\right)$, there exists $g \in H$ which is not \pm 1 , $\pm g_{0}$. If

$$
g=\left(\begin{array}{cc}
a & b \\
c & -a
\end{array}\right) \in H
$$

is such an element, we have $a \neq 0$, since otherwise $b=-c^{-1}$ and the trace of $g_{0} g$ is $c+c^{-1}$, which is not in $\{-2,0,2\}$ (nonzero because -1 is not a square in our first subcase), so $H_{\text {nreg }}^{(2)} \neq \emptyset$, contrary to the assumption. Moreover, we can find $g$ as above with $b \neq c$ : otherwise, it would follow that $H$ is contained in the normalizer of a nonsplit maximal torus, again contradicting the assumption that $H$ is a generating set. 
Now we argue with $g$ as above (i.e., $a \neq 0, b \neq c$ ). We have

$$
g_{0} g=\left(\begin{array}{cc}
c & -a \\
-a & -b
\end{array}\right) \in H^{(2)} \text {, }
$$

with nonzero trace $t=c-b$. Moreover, if $t=2$, that is, $c=b+2$, the condition $\operatorname{det}\left(g_{0} g\right)=1$ implies

$$
-2 b-b^{2}-a^{2}=1
$$

or $(b+1)^{2}=-a^{2}$. Similarly, if $t=-2$, we obtain $(b-1)^{2}=-a^{2}$. Since $a \neq 0$, it follows in both cases that -1 is a square in $\mathbf{F}_{p}$, which contradicts our assumption in the first subcase.

Now we come to the second subcase when $-1=z^{2}$ is a square in $\mathbf{F}_{p}$. We can then diagonalize $g_{0}$ over $\mathbf{F}_{p}$, and conjugating again, this means we can assume that $H$ contains

$$
g_{0}^{\prime}=\left(\begin{array}{cc}
z & 0 \\
0 & -z
\end{array}\right)
$$

as well as some other matrix

$$
g^{\prime}=\left(\begin{array}{cc}
a & b \\
c & -a
\end{array}\right)
$$

(the values of $a, b$, and $c$ are not the same as before; we are still in the case when every element of $H$ has trace 0 except for \pm 1 ).

Now the trace of $g_{0}^{\prime} g^{\prime} \in H^{(2)}$ is $2 z a$. But we can find $g^{\prime}$ with $a \neq 0$, since otherwise $H$ would again not be a generating set, being contained in the normalizer of the diagonal (split) maximal torus, and so this trace is nonzero.

The condition $2 z a= \pm 2$ would give $z a= \pm 1$, which leads to $-a^{2}=1$. But since $1=\operatorname{det}\left(g^{\prime}\right)=-a^{2}-b c$, we then obtain $b c=0$ for all elements of $H$. Finally, if all elements of $H$ satisfy $b=0$, the set $H$ would be contained in the subgroup of upper-triangular matrices. So we can find a matrix in $H$ with $b \neq 0$, hence $c=0$. Similarly, we can find another

$$
g^{\prime \prime}=\left(\begin{array}{cc}
a & 0 \\
c & -a
\end{array}\right)
$$

in $H$ with $c \neq 0$. Taking into account that $z^{2}=-1$, computing the traces of $g^{\prime} g^{\prime \prime}$ and of $g_{0}^{\prime} g^{\prime} g^{\prime \prime}$ gives

$$
b c-2, \quad b c z,
$$


respectively. If $b c=2$, the third trace (of an element in $H^{(3)}$ ) is $2 z \notin\{0,2,-2\}$ since $p \neq 2$, and if $b c=4$, it is $4 z \notin\{0,2,-2\}$ since $p \neq 5$. And of course if $b c \notin\{2,4\}$, the first trace is already not in $\{-2,0,2\}$. So we are done.

For the proof of Theorem 3.11, we will use the method suggested by Larsen and Pink at the beginning of [17, Section 4]. We consider the map

$$
\phi\left\{\begin{array}{l}
\operatorname{Cl}(g) \times \operatorname{Cl}(g) \times \operatorname{Cl}(g) \longrightarrow \mathrm{G} \times \mathrm{G}, \\
\left(x_{1}, x_{2}, x_{3}\right) \mapsto\left(x_{1} x_{2}, x_{1} x_{3}\right)
\end{array}\right.
$$

and we note that for $\left(x_{1}, x_{2}, x_{3}\right) \in(\operatorname{Cl}(g) \cap H)^{3}$, we have $\phi\left(x_{1}, x_{2}, x_{3}\right) \in H^{(2)} \times H^{(2)}$. We then hope that the fibers $\phi^{-1}\left(y_{1}, y_{2}\right)$ of $\phi$ are all finite with size bounded independently of $\left(y_{1}, y_{2}\right) \in \mathbf{G} \times \mathbf{G}$, say of size at most $c_{1} \geq 1$. The reason behind this hope is that $\operatorname{Cl}(g)^{3}$ and $\mathrm{G}^{2}$ have the same dimension, and hence unless something special happens, we would expect the fibers to have dimension 0, which corresponds to having fibers of bounded size since everything is defined using polynomial equations.

If this hope turns out to be justified, we can count $|\mathbf{C l}(g) \cap H|$ by summing according to the values of $\phi$ : denoting $Z=(\mathbf{C l}(g) \cap H)^{3}$ and $W=\phi(Z)=\phi\left((\mathbf{C l}(g) \cap H)^{3}\right)$, we have

$$
|\mathbf{C l}(g) \cap H|^{3}=|Z|=\sum_{\left(y_{1}, y_{2}\right) \in W}\left|\phi^{-1}\left(y_{1}, y_{2}\right) \cap Z\right|
$$

which-under our optimistic assumption-leads to the estimate

$$
|\mathrm{Cl}(g) \cap H|^{3} \leq C_{1}|W| \leq C_{1}\left|H^{(2)}\right|^{2} \leq C_{1} \alpha^{2}|H|
$$

which has the form we want.

To implement this - and solve the complications that arise-we are led to analyze the fibers of the map $\phi$. The resulting computations were explained to the author by R. Pink, and start with an easy observation:

Lemma 3.13. Let $k$ be any field, and let $G=\mathrm{SL}_{2}(k)$. Let $C \subset G$ be a conjugacy class, and define

$$
\phi\left\{\begin{array}{l}
C^{3} \longrightarrow G^{2} \\
\left(x_{1}, x_{2}, x_{3}\right) \mapsto\left(x_{1} x_{2}, x_{1} x_{3}\right) .
\end{array}\right.
$$


Then for any $\left(y_{1}, y_{2}\right) \in G \times G$, we have a bijection

$$
\begin{aligned}
C \cap y_{1} C^{-1} \cap y_{2} C^{-1} & \longrightarrow \phi^{-1}\left(y_{1}, y_{2}\right), \\
x_{1} & \mapsto\left(x_{1}, x_{1}^{-1} y_{1}, x_{1}^{-1} y_{2}\right) .
\end{aligned}
$$

In particular, if $k=\overline{\mathbf{F}}_{p}$ and $C$ is a regular semisimple conjugacy class, we have a bijection

$$
\phi^{-1}\left(y_{1}, y_{2}\right) \longrightarrow C \cap y_{1} C \cap y_{2} C
$$

Proof. Taking $x_{1}$ as a parameter, any $\left(x_{1}, x_{2}, x_{3}\right)$ with $\phi\left(x_{1}, x_{2}, x_{3}\right)=\left(y_{1}, y_{2}\right)$ can certainly be written $\left(x_{1}, x_{1}^{-1} y_{1}, x_{1}^{-1} y_{2}\right)$. Conversely, such an element in $\mathrm{SL}_{2}(k)^{3}$ really belongs to $C^{3}$ (hence to the fiber) if and only if $x_{1} \in C, x_{1}^{-1} y_{1} \in C, x_{1}^{-1} y_{2} \in C$, that is, if and only if $x_{1} \in C \cap y_{1} C^{-1} \cap y_{2} C^{-1}$, which proves the first part.

For the second part, we need only notice that if $C$ is a regular semisimple conjugacy class, say that of $g$, then $C=C^{-1}$ because $g^{-1}$ has the same characteristic polynomial as $g$, hence is conjugate to $g$.

We are now led to determine when an intersection of the form $C \cap y_{1} C \cap y_{2} C$ can be infinite. The answer is as follows, and it is one place where the use of the infinite group $\mathrm{SL}_{2}\left(\overline{\mathbf{F}}_{p}\right)$ is significant:

Lemma 3.14 (Pink). Let $k$ be an algebraically closed field of characteristic not equal to 2 , and let $g \in \mathrm{SL}_{2}(k)$ be a regular semisimple element, $C$ the conjugacy class of $g$. For $y_{1}, y_{2} \in G$, the intersection $X=C \cap y_{1} C \cap y_{2} C$ is finite, containing at most two elements, unless one of the following cases holds:

(1) We have $y_{1}=1$, or $y_{2}=1$ or $y_{1}=y_{2}$.

(2) There exists a conjugate $\mathbf{B}=x \mathbf{B}_{0} X^{-1}$ of the subgroup

$$
\mathbf{B}_{0}=\left\{\left(\begin{array}{cc}
a & b \\
0 & a^{-1}
\end{array}\right)\right\} \subset \mathrm{SL}_{2}(k)
$$

and an element $t \in \mathbf{B} \cap C$ such that

$$
y_{1}, y_{2} \in \mathbf{U} \cup t^{2} \mathbf{U}
$$


where

$$
\mathrm{U}=x \mathrm{U}_{0} x^{-1}, \quad \mathrm{U}_{0}=\left\{\left(\begin{array}{ll}
1 & b \\
0 & 1
\end{array}\right)\right\} \subset \mathbf{B}_{0}
$$

In that case, we have $X \subset C \cap \mathbf{B}$.

(3) The trace of $g$ is 0.

The proof will be given at the end of this section: it is mostly computational. Before coming back to the proof of Theorem 3.11, we state and prove another preliminary lemma, which is another case of nonconcentration inequalities.

Lemma 3.15. For a prime $p$ and $\gamma \in \overline{\mathbf{F}}_{p}^{\times}$, define

$$
C_{\gamma}=\left\{\left(\begin{array}{cc}
\gamma & t \\
0 & \gamma^{-1}
\end{array}\right) \mid t \in \overline{\mathbf{F}}_{p}\right\}
$$

For any $p \geq 3$, any $\gamma \in \overline{\mathbf{F}}_{p}^{\times}$, any $x \in \mathrm{SL}_{2}\left(\overline{\mathbf{F}}_{p}\right)$ and any symmetric generating set $H$ of $\mathrm{SL}_{2}\left(\mathbf{F}_{p}\right)$ containing 1 , we have

$$
\left|H \cap x C_{\gamma} x^{-1}\right|=\left|H \cap x\left\{\left(\begin{array}{cc}
\gamma & t \\
0 & \gamma^{-1}
\end{array}\right) \mid t \in \overline{\mathbf{F}}_{p}\right\} x^{-1}\right| \leq 2 \alpha^{2}|H|^{1 / 3},
$$

where $\alpha=\operatorname{trp}(H)$.

Proof. We first deal with the fact that $x$ and $\gamma$ are not necessarily in $\operatorname{SL}_{2}\left(\mathbf{F}_{p}\right)$. We have $x C_{\gamma} x^{-1} \cap \mathrm{SL}_{2}\left(\mathbf{F}_{p}\right) \subset x \mathbf{B}_{0} x^{-1} \cap \mathrm{SL}_{2}\left(\mathbf{F}_{p}\right)$, and there are three possibilities for the latter: either $x \mathbf{B}_{0} X^{-1} \cap \mathrm{SL}_{2}\left(\mathbf{F}_{p}\right)=1$, or $x \mathbf{B}_{0} X^{-1} \cap \mathrm{SL}_{2}\left(\mathbf{F}_{p}\right)=T$ is a nonsplit maximal torus of $\mathrm{SL}_{2}\left(\mathbf{F}_{p}\right)$, or $x \mathbf{B}_{0} X^{-1} \cap \mathrm{SL}_{2}\left(\mathbf{F}_{p}\right)=B$ is an $\mathrm{SL}_{2}\left(\mathbf{F}_{p}\right)$-conjugate of the group $B_{0}=\mathbf{B}_{0} \cap \mathrm{SL}_{2}\left(\mathbf{F}_{p}\right)$ of upper-triangular matrices (this is once more a standard property of linear algebraic groups over finite fields; the most direct argument in this special case is probably to observe that we only need to know that $x \mathbf{B}_{0} X^{-1} \cap \mathrm{SL}_{2}\left(\mathbf{F}_{p}\right)$ is a subset of a maximal torus, or of a conjugate of $B$, which follows from the fact that this intersection is a solvable subgroup of $\mathrm{SL}_{2}\left(\mathbf{F}_{p}\right)$ ).

In the last case, we can assume that $x \in \mathrm{SL}_{2}\left(\mathbf{F}_{p}\right)$ and $\gamma \in \mathbf{F}_{p}$. In the first, of course, there is nothing to do. And as for the second, note that $\gamma$ and $\gamma^{-1}$ are the eigenvalues of any element in $\mathrm{SL}_{2}\left(\mathrm{~F}_{p}\right) \cap x C_{\gamma} x^{-1}$, and there are at most two elements in a maximal torus with given eigenvalues. A fortiori, we have $\left|H \cap x C_{\gamma} X^{-1}\right| \leq 2 \leq 2 \alpha^{2}|H|^{1 / 3}$ in that case. 
Thus, we are left with the situation where $x \in \mathrm{SL}_{2}\left(\mathbf{F}_{p}\right)$. Using $\mathrm{SL}_{2}\left(\mathbf{F}_{p}\right)$-conjugation, it is enough to deal with the case $x=1$. Then either the intersection is empty (and the result is true) or we can fix

$$
g_{0}=\left(\begin{array}{cc}
\gamma & t_{0} \\
0 & \gamma^{-1}
\end{array}\right) \in H \cap C_{\gamma},
$$

and observe that for any $g \in H \cap C_{\gamma}$, we have

$$
g_{0}^{-1} g \in H^{(2)} \cap C_{1},
$$

hence

$$
\left|H \cap C_{\gamma}\right| \leq\left|H^{(2)} \cap C_{1}\right|=\left|H^{(2)} \cap \mathrm{U}_{0}\right|,
$$

which reduces further to the case $\gamma=1$.

In that case, we have another case of the Larsen-Pink nonconcentration inequality, in that case in a one-dimensional variety. Here also, we can give a rather short proof: we fix any element $h \in H$ such that $h$ is not in $\mathbf{B}_{0}$, that is,

$$
h=\left(\begin{array}{ll}
a & b \\
c & d
\end{array}\right)
$$

with $c \neq 0$. This element exists, because otherwise $H \subset \mathbf{B} \cap \mathrm{SL}_{2}\left(\mathbf{F}_{p}\right)$ would not be a generating set of $\mathrm{SL}_{2}\left(\mathbf{F}_{p}\right)$.

Now, consider the multiplication map

$$
\psi:\left\{\begin{array}{l}
\mathbf{U}^{*} \times \mathbf{U}^{*} \times \mathbf{U}^{*} \longrightarrow \mathbf{G}, \\
\left(u_{1}, u_{2}, u_{3}\right) \mapsto u_{1} h u_{2} h^{-1} u_{3},
\end{array}\right.
$$

where $\mathrm{U}^{*}=\mathrm{U}_{0}-1$ (we explain below why we do not use $\mathrm{U}_{0}^{3}$ as the domain).

Note that since $h \in H$, we have $\psi\left(\left(\mathbf{U}^{*} \cap H^{(2)}\right)^{3}\right) \subset H^{(8)}$. Crucially, we claim that for any $x \in \mathrm{G}$, the fiber $\psi^{-1}(x)$ is either empty or reduced to a single element! If this is true, we obtain as before

$$
\left|\mathrm{U}^{*} \cap H^{(2)}\right|^{3} \leq\left|H^{(8)}\right| \leq \alpha^{6}|H|,
$$

and therefore

$$
\left|\mathrm{U}_{0} \cap H^{(2)}\right|=\left|\mathrm{U}^{*} \cap H^{(2)}\right|+1 \leq 2 \alpha^{2}|H|^{1 / 3},
$$

which is the result. 
To check the claim, we compute. Precisely, if

$$
u_{i}=\left(\begin{array}{cc}
1 & t_{i} \\
0 & 1
\end{array}\right) \in \mathbf{U}^{*}
$$

a matrix multiplication leads to

$$
\psi\left(u_{1}, u_{2}, u_{3}\right)=\left(\begin{array}{cc}
1-t_{1} t_{2} c^{2}-t_{2} a c & \star \\
-t_{2} c^{2} & \star
\end{array}\right),
$$

and in order for this to be a fixed matrix $x$, we see that $t_{2}$ (i.e., $u_{2}$ ) is uniquely determined (since $c \neq 0$ ). Since $u_{2}$ is in $U^{*}$, it is not 1 , and this means that $t_{2} \neq 0$ (ensuring this is the reason that $\psi$ is defined using $\mathbf{U}^{*}$ instead of $\mathbf{U}_{0}$ ). Thus $t_{1}$ (i.e., $u_{1}$ ) is also uniquely determined, and finally

$$
u_{3}=\left(u_{1} h u_{2} h^{-1}\right)^{-1} X
$$

is uniquely determined.

Proof of Theorem 3.11. We have $g$ regular semisimple with $\operatorname{Tr}(g) \neq 0$. We define as above the map $\phi$ and denote

$$
Z=(\mathbf{C l}(g) \cap H)^{3}, \quad W=\phi(Z)=\phi\left((\mathbf{C l}(g) \cap H)^{3}\right),
$$

so that

$$
|\mathbf{C l}(g) \cap H|^{3}=\sum_{\left(y_{1}, y_{2}\right) \in W}\left|\phi^{-1}\left(y_{1}, y_{2}\right) \cap Z\right|=S_{0}+S_{1}+S_{2},
$$

where $S_{i}$ denotes the sum restricted to a subset $W_{i} \subset W, W_{0}$ being the subset where the fiber has order at most 2, while $W_{1}$ and $W_{2}$ correspond to those $\left(y_{1}, y_{2}\right)$ where cases (1) and (2) of Lemma 3.14 hold. Precisely, we do not put into $W_{2}$ the $\left(y_{1}, y_{2}\right)$ for which both cases (1) and (2) are valid, for example, $y_{1}=1$, and we add to $W_{1}$ the cases where $y_{1}=-1$, which may otherwise appear in Case (2). We will prove:

$$
S_{0} \leq 2\left|H^{(2)}\right|^{2} \leq 2 \alpha^{2}|H|^{2}, \quad S_{1} \leq 4\left|H^{(2)}\right|^{2} \leq 4 \alpha^{2}|H|^{2}, \quad S_{2} \leq 32 \alpha^{34 / 3}|H|^{5 / 3} .
$$

Assuming this, we immediately obtain

$$
|\mathbf{C l}(g) \cap H| \leq 6^{2 / 3} \alpha^{2 / 3}|H|^{2 / 3}+2^{5 / 3} \alpha^{34 / 9}|H|^{5 / 9}
$$


from (3.9). Now either the second term is smaller than the first, and we obtain (3.3) (since $\left.2 \cdot 6^{2 / 3}<7\right)$, or

$$
2^{5 / 3} \alpha^{34 / 9}|H|^{5 / 9}>6^{2 / 3} \alpha^{2 / 3}|H|^{2 / 3}>2^{5 / 3} \alpha^{2 / 3}|H|^{2 / 3},
$$

which gives

$$
\alpha>|H|^{1 / 28}
$$

the second alternative (3.4) of Theorem 3.11, which is therefore proved.

We now check the bounds on $S_{i}$. The case of $S_{0}$ follows by the fact that the fibers over $W_{0}$ have at most two elements, hence also their intersection with $Z$, and that $\left|W_{0}\right| \leq$ $|W| \leq\left|H^{(2)}\right|^{2}$.

The case of $S_{1}$ splits into four almost identical subcases, corresponding to $y_{1}=1$, $y_{1}=-1$ (remember that we added this, borrowing it from Case (2)), $y_{2}=1$ or $y_{1}=y_{2}$. We deal only with the first, say $S_{1,1}$ : we have

$$
S_{1,1} \leq \sum_{y_{2} \in H^{(2)}}\left|\phi^{-1}\left(1, y_{2}\right) \cap Z\right| .
$$

But using Lemma 3.13, we have

$$
\left|\phi^{-1}\left(1, y_{2}\right) \cap Z\right|=\left|\left\{\left(x_{1}, x_{1}^{-1}, x_{1}^{-1} y_{2}\right) \in(\mathbf{C l}(g) \cap H)^{3}\right\}\right| \leq\left|H^{(3)}\right|
$$

for any given $y_{2} \in H^{(2)}$, since $x_{1} \in H$ determines the triple $\left(x_{1}, x_{1}^{-1}, x_{1}^{-1} y_{2}\right)$. Therefore,

$$
S_{1,1} \leq\left|H^{(2)}\right||H| \leq\left|H^{(2)}\right|^{2}
$$

and similarly for the other three cases.

Now for $S_{2}$. Here also we sum over $y_{1}$ first, which is $\neq \pm 1$ (by our definition of $W_{2}$ ). The crucial point is then that an element $y_{1} \neq \pm 1$ is included in at most two conjugates of $\mathbf{B}_{0}$. Hence, up to a factor 2, the choice of $y_{1}$ fixes that of the relevant conjugate $\mathbf{B}$ for which Case (2) applies. Next we observe that $C_{\mathrm{B}}=\mathbf{C l}(g) \cap \mathrm{B}$ is a conjugate of the union

$$
C_{\alpha} \cup C_{\alpha^{-1}}
$$

where, as in Lemma 3.15, we define

$$
C_{\alpha}=\left\{\left(\begin{array}{cc}
\alpha & t \\
0 & \alpha^{-1}
\end{array}\right) \mid t \in \overline{\mathbf{F}}_{p}\right\}
$$


and $\alpha$ is such that $\alpha+\alpha^{-1}=\operatorname{Tr}(g)$. Given $y_{1} \in H^{(2)}$ and B containing $y_{1}$, we have by (3.8)

$$
y_{2} \in\left(H^{(2)} \cap \mathbf{U}\right) \cup\left(H^{(2)} \cap t^{2} \mathbf{U}\right)
$$

for some $t \in C_{\mathrm{B}}$. We note that $t^{2} \mathrm{U}$ is itself conjugate to $C_{\alpha^{2}}$ or $C_{\alpha^{-2}}$.

Then the size of the fiber $\phi^{-1}\left(y_{1}, y_{2}\right) \cap Z$ is determined by the number of possibilities for $x_{1}$. As the latter satisfies

$$
x_{1} \in C_{\mathrm{B}} \cap H,
$$

we see that we must estimate the size of intersections of the type

$$
H \cap C_{\gamma}, \quad H^{(2)} \cap C_{\gamma}
$$

for some fixed $\gamma \in \mathbf{F}_{p}^{\times}$, as this will lead us to estimates for the number of possibilities for $y_{2}$ as well as $x_{1}$. Using Lemma 3.15 twice, we obtain

$$
\left|\left\{y_{2} \mid\left(y_{1}, y_{2}\right) \in W_{2}\right\}\right| \leq 8 \operatorname{trp}\left(H^{(2)}\right)^{2}\left|H^{(2)}\right|^{1 / 3} \leq 8 \alpha^{25 / 3}|H|^{1 / 3},
$$

(the factor 8 accounts for the two possible choices of $\mathbf{B}$ and the two "components" for $y_{2}$, and the factor 2 in the lemma) and

$$
\left|\phi^{-1}\left(y_{1}, y_{2}\right) \cap Z\right| \leq 4 \alpha^{2}|H|^{1 / 3} .
$$

This gives

$$
S_{2} \leq 32 \alpha^{31 / 3}|H|^{2 / 3}\left|H^{(2)}\right| \leq 32 \alpha^{34 / 3}|H|^{5 / 3},
$$

as claimed.

There now only remains to prove Lemma 3.14.

Proof of Lemma 3.14. It will be convenient to compute the intersection $C \cap y_{1}^{-1} C \cap$ $y_{2}^{-1} C$ instead of $C \cap y_{1} C \cap y_{2} C$, a change of notation which is innocuous.

The computation is then based on a list of simple checks. We can assume that the regular semisimple element $g$ is

$$
g=\left(\begin{array}{cc}
\alpha & 0 \\
0 & \alpha^{-1}
\end{array}\right)
$$


where $\alpha^{4} \neq 1$, because $\alpha= \pm 1$ implies that $g$ is not regular semisimple, and $\alpha$ a fourth root of unity implies that $\operatorname{Tr}(g)=0$, which is the third case of the lemma (recall that $k$ is assumed to be algebraically closed). Thus, the conjugacy class $C$ is the set of matrices of trace equal to $t=\alpha+\alpha^{-1}$.

The only trick involved is that, for any $y_{1} \in \mathrm{SL}_{2}(k)$ and $x \in \mathrm{SL}_{2}(k)$, we have

$$
C \cap\left(x y_{1} x^{-1}\right)^{-1} C=x\left(x^{-1} C \cap y_{1}^{-1} x^{-1} C\right)=x\left(C \cap Y_{1}^{-1} C\right) x^{-1}
$$

since $X^{-1} C=C x^{-1}$, by definition of conjugacy classes. This means that we can compute $C \cap y_{1}^{-1} C$, up to conjugation, by looking at $C \cap\left(y_{1}^{\prime}\right)^{-1} C$ for any $y_{1}^{\prime}$ in the conjugacy class of $y_{1}$. In particular, of course, determining whether $C \cap y_{1}^{-1} C$ is infinite or not only depends on the conjugacy class of $Y_{1}$.

The conjugacy classes in $\mathrm{SL}_{2}(k)$ are well known. We will run through representatives of these classes in order, and determine the corresponding intersection $C \cap Y_{1}^{-1} C$. Then, to compute $C \cap y_{1}^{-1} C \cap y_{2}^{-1} C$, we take an element $x$ in $C \cap Y_{1}^{-1} C$, compute $y_{2} x$, and $C \cap y_{1}^{-1} C \cap y_{2}^{-1} C$ corresponds to those $x$ for which the trace of $y_{2} X$ is also equal to $t$.

We assume $y_{1} \neq \pm 1$. Then we distinguish four cases:

$$
\begin{aligned}
& y_{1}=\left(\begin{array}{ll}
1 & 1 \\
0 & 1
\end{array}\right), \quad y_{1}=\left(\begin{array}{cc}
-1 & 1 \\
0 & -1
\end{array}\right), \\
& y_{1}=\left(\begin{array}{cc}
\beta & 0 \\
0 & \beta^{-1}
\end{array}\right), \quad \beta \neq \pm 1, \beta \neq \alpha^{ \pm 2}, \\
& y_{1}=\left(\begin{array}{cc}
\alpha^{2} & 0 \\
0 & \alpha^{-2}
\end{array}\right) .
\end{aligned}
$$

We claim that $D=C \cap y_{1}^{-1} C$ is then given, respectively, by the sets containing all matrices of the following forms, parameterized by an element $a \in k$ (with $a \neq 0$ in the third case):

$$
\begin{aligned}
& \left(\begin{array}{cc}
\alpha & a \\
0 & \alpha^{-1}
\end{array}\right) \text { or }\left(\begin{array}{cc}
\alpha^{-1} & a \\
0 & \alpha
\end{array}\right), \\
& \left(\begin{array}{cc}
a & \left(-a^{2}+a t-1\right) /(2 t) \\
2 t & t-a
\end{array}\right)
\end{aligned}
$$




$$
\begin{aligned}
& \frac{1}{\beta+1}\left(\begin{array}{cc}
t & \left(\beta-\alpha^{2}\right) a \\
-\left(\beta-\alpha^{-2}\right) a^{-1} & t \beta
\end{array}\right), \\
& \left(\begin{array}{cc}
\alpha^{-1} & a \\
0 & \alpha
\end{array}\right) \text { or }\left(\begin{array}{cc}
\alpha^{-1} & 0 \\
a & \alpha
\end{array}\right) .
\end{aligned}
$$

Let us check, for instance, the third and fourth cases (cases (1) and (2) are left as exercise), which we can do simultaneously, taking $y_{1}$ as in (3.10) but without assuming $\beta \neq \alpha^{ \pm 2}$. For

$$
x=\left(\begin{array}{ll}
a & b \\
c & d
\end{array}\right) \in C,
$$

we compute

$$
y_{1} X=\left(\begin{array}{cc}
\beta a & \beta b \\
\beta^{-1} c & \beta^{-1} d
\end{array}\right) .
$$

This matrix belongs to $C$ if and only if $\beta a+\beta^{-1} d=t=a+d$. This means that $(a, d)$ is a solution of the linear system

$$
\begin{array}{r}
a+d=t, \\
\beta a+\beta^{-1} d=t,
\end{array}
$$

of determinant $\beta^{-1}-\beta \neq 0$, so that we have

$$
a=\frac{t}{\beta+1}, \quad d=\frac{\beta t}{\beta+1} .
$$

Write $c=c^{\prime} /(\beta+1), \quad b=b^{\prime} /(\beta+1)$; then the condition on $c^{\prime}$ and $b^{\prime}$ to have $\operatorname{det}(x)=1$ can be expressed as

$$
-c^{\prime} b^{\prime}=\left(\beta-\alpha^{2}\right)\left(\beta-\alpha^{-2}\right) .
$$

This means that either $\beta$ is not one of $\alpha^{2}, \alpha^{-2}$ (the third case), and then $c$ and $d$ are nonzero, and we can parametrize the solutions as in (3.12), or else (the fourth case) $c$ or $d$ must be zero, and then we obtain upper or lower-triangular matrices, as described in (3.13).

Now we intersect $D$ (in the general case again) with $y_{2}^{-1} C$. We write

$$
y_{2}=\left(\begin{array}{ll}
x_{1} & x_{2} \\
x_{3} & x_{4}
\end{array}\right)
$$


We consider the first of our four possibilities now, so that $x \in D$ is uppertriangular with diagonal coefficients $\alpha, \alpha^{-1}$ (as a set), see (3.11). We compute the trace of $y_{2} x$, and find that is

$$
a x_{3}+x_{1} \alpha+x_{4} \alpha^{-1} \text { or } a x_{3}+x_{1} \alpha^{-1}+x_{4} \alpha
$$

Thus, if $x_{3} \neq 0$, there is at most one value of $a$ for which the trace is $t$, that is, $D \cap y_{2}^{-1} C$ has at most two elements (one for each form of the diagonal). If $x_{3}=0$, we find that $x_{1}$ is a solution of

$$
\alpha x_{1}+\alpha^{-1} x_{1}^{-1}=t
$$

or

$$
\alpha x_{1}^{-1}+\alpha^{-1} x_{1}=t
$$

for which the solutions are among $1, \alpha^{2}$, and $\alpha^{-2}$, so that $y_{2}$ is upper-triangular with diagonal coefficients $(1,1),\left(\alpha^{2}, \alpha^{-2}\right)$, or $\left(\alpha^{-2}, \alpha^{2}\right)$, and this is one of the instances of Case (2) of Lemma 3.14.

Let us now consider the second of our four cases, leaving this time the third and fourth to the reader. Thus, we take $x$ as in (3.12), and compute the trace of $y_{2} x$ as a function of $a$, which gives

$$
\operatorname{Tr}\left(y_{2} x\right)=-\frac{x_{3}}{2 t} a^{2}+\left(x_{1}-x_{4}+\frac{x_{3}}{2}\right) a+\left(x_{4}+2 x_{2}\right) t
$$

The equation $\operatorname{Tr}\left(y_{2} x\right)=t$ has therefore at most two solutions, unless $x_{3}=0$ and $x_{4}=x_{1}$. In that case, we have $x_{4}=1$, and the constant term is equal to $t$ if and only if $x_{4}=1$ and $x_{2}=0$ (so $y_{2}=1$ ) or $x_{4}= \pm 1$ and $x_{2}=1$ (and then $y_{2}=y_{1}$ ). Each of these possibilities corresponds to the exceptional situation of Case (1) of Lemma 3.14.

Checking similarly the remaining cases, we finish the proof.

\subsection{Proof of Helfgott's Theorem}

We now prove Theorem 1.2. If $p \leq 5$, one checks numerically that trivial bounds already imply the theorem. So we assume that $p \geq 7$, which means that Lemma 3.10 is applicable. We will show that

$$
\operatorname{trp}(H) \geq 2^{-1 / 2}|H|^{1 / 1512}
$$


for $p \geq 7$, unless $H^{(3)}=\mathrm{SL}_{2}\left(\mathbf{F}_{p}\right)$, where the latter case will arise by applying Proposition 3.9. Then using Lemma 3.2, we derive

$$
\operatorname{trp}(H) \geq \max \left(2^{1 / 2}, 2^{-1 / 2}|H|^{1 / 1512}\right) \geq|H|^{1 / 3024},
$$

which is the precise form of Helfgott's Theorem we claimed.

We define $\tilde{H}=H^{(2)}$, so that (by Lemma 3.10) there exists at least one maximal torus T involved with $H^{(3)}$, hence a fortiori involved with $L=\tilde{H}^{(2)}=H^{(4)}$.

If, among all maximal tori involved with $L$, there is one for which the lower bound (3.5) (applied to $H=L$ ) fails, we obtain from Proposition 3.12 the lower bound

$$
\operatorname{trp}(L) \geq|L|^{1 / 168} \geq|H|^{1 / 168}
$$

and since $\operatorname{trp}(L) \leq \alpha^{9}$ by Ruzsa's Lemma, we obtain

$$
\alpha \geq|H|^{1 / 1512} \geq 2^{-1 / 2}|H|^{1 / 1512}
$$

which is (3.14).

Otherwise, we distinguish two cases.

Case (1). There exists a maximal torus $\mathrm{T}$ involved with $L$ such that, for any $g \in G$, the torus $g \mathrm{~T}^{-1}$ is involved with $L$.

As we can guess from (3.5) and (3.2), in that case, the set $L$ will tend to be rather large, so $|L|$ is close to $|G|$, unless the tripling constant is itself large enough.

Precisely, writing $T=\mathbf{T} \cap G$, we note that the maximal tori

$$
g T g^{-1}=\left(g \mathbf{T} g^{-1}\right) \cap G
$$

are distinct for $g$ taken among representatives of $G / N_{G}(T)$. Then we have the inequalities

$$
\left|L^{(2)}\right| \geq \sum_{g \in G / N_{G}(T)}\left|L^{(2)} \cap g \mathbf{T}_{\mathrm{reg}} g^{-1}\right| \geq 7^{-1} \beta^{-4}|L|^{1 / 3} \frac{|G|}{\left|N_{G}(T)\right|}
$$

where $\beta=\operatorname{trp}(L)$, since each $g \mathbf{T} g^{-1}$ is involved with $L$ and distinct regular semisimple elements lie in distinct maximal tori (and we are in a case where (3.5) holds for all tori involved with $L$ ). 
Now we unwind this inequality in terms of $H$ and $\alpha=\operatorname{trp}(H)$. We have $L^{(2)}=H^{(8)}$, so

$$
|H| \geq \alpha^{-6}\left|L^{(2)}\right| \geq 14^{-1} \alpha^{-6} \beta^{-4}(p-1)^{2}|L|^{1 / 3} \geq 14^{-1} \alpha^{-6} \beta^{-4}(p-1)^{2}|H|^{1 / 3}
$$

by Ruzsa's Lemma. Furthermore, we have

$$
\beta=\operatorname{trp}(L)=\operatorname{trp}\left(H^{(4)}\right) \leq \alpha^{9}
$$

by Ruzsa's Lemma again, and hence the inequality gives the bound

$$
|H| \geq 14^{-3 / 2} \alpha^{-63}(p-1)^{3},
$$

which for $p \geq 5$ implies $|H| \geq 100^{-1} \alpha^{-63}|G|$. But then either

$$
\operatorname{trp}(H)=\alpha \geq 200^{-1 / 63}|G|^{1 / 567} \geq 2^{-1 / 2}|H|^{1 / 621},
$$

or else

$$
|H| \geq 2|G|^{8 / 9},
$$

which (via Proposition 3.9) are versions of the two alternatives we are seeking (in particular, the first implies (3.14).)

Case (2). Since we know that some torus is involved with $L$, the complementary situation to Case (1) is that there exists a maximal torus T involved with $L=H^{(4)}$ and a conjugate $g \mathbf{T} g^{-1}$, for some $g \in G$, which is not involved with $L$. We are then going to obtain growth using Lemma 3.5. There is a first clever observation (the idea of which goes back to work of Glibichuk and Konyagin [9] on the "sum-product phenomenon"): one can assume, possibly after changing $\mathbf{T}$ and $g$, that $g$ is in $H$.

Indeed, to check this claim, we start with $\mathbf{T}$ and $h$ as above. Since $H$ is a generating set, we can write

$$
g=h_{1} \cdots h_{m}
$$

for some $m \geq 1$ and some elements $h_{i} \in H$. Now let $i \leq m$ be the smallest index such that the maximal torus

$$
\mathbf{T}^{\prime}=\left(h_{i+1} \cdots h_{m}\right) \mathbf{T}\left(h_{i+1} \cdots h_{m}\right)^{-1}
$$


is involved with $L$. Taking $i=m$ means that $\mathbf{T}$ is involved with $L$, which is the case, and therefore the index $i$ exists. Moreover $i \neq 0$, again by definition. It follows that

$$
\left(h_{i} h_{i+1} \cdots h_{m}\right) \mathbf{T}\left(h_{i} h_{i+1} \cdots h_{m}\right)^{-1}
$$

is not involved with $L$. But this means that we can replace $(\mathbf{T}, g)$ with $\left(\mathbf{T}^{\prime}, h_{i}\right)$, and since $h_{i} \in H$, this gives us the claim.

We now write $h$ for the conjugator $g$ such that $L$ and the torus $\mathbf{S}=g \mathbf{T} g^{-1}=h \mathbf{T} h^{-1}$ are not involved. Apply Lemma 3.5 with $(H, K)=\left(\tilde{H}, h \mathbf{T} h^{-1} \cap G\right)$ and $n=5$. This gives

$$
\frac{\left|\tilde{H}^{(6)}\right|}{|\tilde{H}|} \geq \frac{\left|\tilde{H}^{(5)} \cap S\right|}{\left|\tilde{H}^{(2)} \cap S\right|} .
$$

But since $L=\tilde{H}^{(2)}$ and $\mathbf{S}$ are not involved (by construction), we have $\left|\tilde{H}^{(2)} \cap S\right| \leq 2$, by the easy part of the Key Proposition 3.12, and therefore

$$
\frac{\left|\tilde{H}^{(6)}\right|}{|\tilde{H}|} \geq \frac{1}{2}\left|\tilde{H}^{(5)} \cap \mathbf{S}\right| .
$$

However, $L$ and $\mathbf{T}$ are involved, and moreover

$$
h\left(H^{(8)} \cap \mathbf{T}\right) h^{-1} \subset H^{(10)} \cap \mathbf{S}=\tilde{H}^{(5)} \cap \mathbf{S},
$$

so that

$$
\left|\tilde{H}^{(5)} \cap \mathbf{S}\right| \geq\left|H^{(8)} \cap T\right|=\left|L^{(2)} \cap T\right| \geq 14^{-1} \beta^{-4}|L|^{1 / 3},
$$

where $\beta=\operatorname{trp}(L)$, by the Key Proposition 3.12 (again, because (3.5) holds for all tori involved with $L$ ).

Thus,

$$
\frac{\left|\tilde{H}^{(6)}\right|}{|\tilde{H}|} \geq 28^{-1} \beta^{-4}|H|^{1 / 3},
$$

which translates to

$$
\alpha^{10}|H| \geq 28^{-1} \alpha^{-36}|H|^{4 / 3}
$$

by Ruzsa's Lemma. This is a rather stronger bound for $\alpha$ than before, namely

$$
\alpha=\operatorname{trp}(H) \geq 28^{-1 / 46}|H|^{1 / 138} \geq 2^{-1 / 2}|H|^{1 / 138}
$$


To summarize, we have obtained three possible lower bounds of the right kind for $\alpha$, namely (3.15), (3.16) and (3.17), one of which holds if $H^{(3)} \neq \mathrm{SL}_{2}\left(\mathbf{F}_{p}\right)$. All imply (3.14), and hence we are done.

\subsection{Diameter bound}

Corollary 1.3 is a well-known consequence of the growth theorem: by induction on $j \geq 1$, we see using Helfgott's Theorem that given a symmetric generating set $S \subset G=\mathrm{SL}_{2}\left(\mathrm{~F}_{p}\right)$, either $\operatorname{diam} \mathcal{C}(G, S) \leq 3^{j}$, or

$$
\left|H^{\left(3^{j}\right)}\right| \geq|H|^{(1+\delta)^{j}}
$$

where $H=S \cup\{1\}$. For

$$
j=\left\lceil\frac{\log \log |G|}{\log (1+\delta)}\right\rceil,
$$

the second alternative is impossible, and hence

$$
\operatorname{diam} \mathcal{C}(G, S) \leq 3^{j} \leq 3(\log |G|)^{(\log 3) / \log (1+\delta)},
$$

which gives the result since $(\log 3) / \log \left(1+\frac{1}{3024}\right) \leq 3323$.

\section{The Bourgain-Gamburd method}

The method of Bourgain and Gamburd [1] leads, from Helfgott's growth theorem, to a proof that the Cayley graphs modulo primes of a Zariski-dense subgroup of $\mathrm{SL}_{2}(\mathbf{Z})$ form an expander family. Applying this method straightforwardly with explicit estimates (as done in [16, Chapter 4]), one obtains explicit expansion bounds (either for the spectral gap of the combinatorial Laplace operator, or for the discrete Cheeger constant). However, these constants are typically very small.

\subsection{The $L^{2}$-flattening inequality}

This section applies_-in principle - to all finite groups, and the basic expansion criterion that we derive (Corollary 4.4, following essentially Bourgain and Gamburd) is also of independent interest.

In rough outline-and probabilistic language - the idea is to show that if two independent $\mathrm{SL}_{2}\left(\mathbf{F}_{p}\right)$-valued symmetrically distributed random variables $X_{1}$ and $X_{2}$ are not too concentrated, but also not very uniformly distributed on $\mathrm{SL}_{2}\left(\mathbf{F}_{p}\right)$, then their product $X_{1} X_{2}$ will be significantly more uniformly distributed, unless there are obvious 
reasons why this should fail to hold. These exceptional possibilities can then be handled separately.

Applying this to some suitable step $X_{k}$ of the random walk (where the initial condition is obtained by different means), this result leads to successive great improvements of the uniformity of the distribution for $X_{2 k}, X_{4 k}, \ldots, X_{2^{j} k}$, until the assumptions of the lemma fail. In that situation, the index $m=2^{j} k$ is of size about $\log |G|$, and $\mathbf{P}\left(X_{2 m}=1\right)$ gives a suitable upper-bound on the number of cycles to obtain expansion, by a variant of what might be called the Huxley-Sarnak-Xue method (see [13,22]), as we now recall.

Remark 4.1. In an earlier draft, we had claimed a much better bound (roughly exponentially better) by using nondyadic steps, but this was due to a bad mistake which was pointed out by the referee, which we heartily thank once more.

For a finite group $G$, we denote by $d(G)$ the minimal dimension of a nontrivial irreducible unitary representation of $G$. Moreover, if $X$ is a $G$-valued symmetrically distributed random variable, we define the return probability $\operatorname{rp}(X)$ by

$$
\operatorname{rp}(X)=\mathbf{P}\left(X_{1} X_{2}=1\right)
$$

where $\left(X_{1}, X_{2}\right)$ are independent random variables with the same distribution as $X$, or equivalently

$$
\operatorname{rp}(X)=\sum_{g \in G} \mathbf{P}(X=g)^{2}
$$

Let $S$ be a symmetric generating subset of $G$ and $\Gamma=\mathcal{C}(G, S)$ the associated Cayley graph. The Markov operator $M$ acts on functions on $G$ by

$$
M \varphi(g)=\frac{1}{|S|} \sum_{s \in S} \varphi(g s)
$$

and it is a self-adjoint operator. The spectral gap of $G$, as we normalize it, is equal to $1-\rho_{\Gamma}^{+}$, where $\rho_{\Gamma}^{+}$is the largest eigenvalue of $M$, and it is therefore $\geq 1-\rho_{\Gamma}$, where $\rho_{\Gamma}$ is the spectral radius of $M$.

By expressing spectrally the number of closed walks of length $2 m$ from the origin in $\Gamma$, and relating the latter with the return probability $\operatorname{rp}\left(X_{m}\right)$, where $\left(X_{m}\right)$ is the 
random walk on the graph governed by $M$, one obtains

$$
\sum_{\rho} \rho^{2 m}=\frac{1}{|G|} \operatorname{rp}\left(X_{m}\right)
$$

Using positivity and the fact that $G$ acts without invariant vector on the $\rho_{\Gamma}$ th eigenspace of $M$, it follows that

$$
d(G) \rho_{\Gamma}^{2 m} \leq \frac{1}{|G|} \operatorname{rp}\left(X_{m}\right)
$$

or in other words, we have a bound for the spectral radius in terms of the return probability: for any $m \geq 1$, we have

$$
1-\lambda_{1}(\Gamma) \leq \rho_{\Gamma} \leq\left(\frac{|G|}{d(G)} \operatorname{rp}\left(X_{m}\right)\right)^{1 /(2 m)}
$$

We consider now two independent (not necessarily identically distributed) $G$ valued random variables $X_{1}$ and $X_{2}$ and let

$$
\operatorname{rp}^{+}\left(X_{1}, X_{2}\right)=\max \left(\operatorname{rp}\left(X_{1}\right), \operatorname{rp}\left(X_{2}\right)\right)
$$

We attempt to bound $\operatorname{rp}\left(X_{1} X_{2}\right)$ in terms of $\operatorname{rp}^{+}\left(X_{1}, X_{2}\right)$. To do this while still remaining at a level of great generality, the following definition will be useful:

Definition 4.2 (Flourishing). For $\delta>0$, a finite group $G$ is $\delta$-flourishing if any symmetric subset $H \subset G$, containing 1, which generates $G$ and has tripling constant $\operatorname{trp}(H)<|H|^{\delta}$ satisfies $H^{(3)}=G$.

In particular, Theorem 1.2 states that all groups $\mathrm{SL}_{2}\left(\mathbf{F}_{p}\right)$, for $p$ prime, are $\frac{1}{3024}-$ flourishing.

We will prove a general $L^{2}$-flattening theorem, which may be of general interest. In order to somehow streamline the proof, we do not explicitly describe here what " $G$ large enough" means. However, all relevant steps where a condition on the size of $G$ occurs are clearly marked, and in the second part of Section 4.3, we will look back to express these as explicit inequalities. 
Theorem 4.3 ( $L^{2}$-flattening conditions). Let $G$ be a finite group which is $\delta$-flourishing for some $\delta$ with $0<\delta \leq 1$. Let $X_{1}$ and $X_{2}$ be symmetric independent $G$-valued random variables.

Let $0<\gamma<1$ be given, and assume that

$$
\mathbf{P}\left(X_{1} \in X H\right) \leq|G|^{-\gamma}
$$

for all proper subgroups $H \subset G$ and all $x \in G$.

Then for any $\varepsilon>0$, there exists $\delta_{1}>0$ and $c_{3}>0$, depending only on $\varepsilon, \delta$, and $\gamma$, such that

$$
\operatorname{rp}\left(X_{1} X_{2}\right) \leq c_{3} \max \left\{\frac{1}{|G|^{1-\varepsilon}}, \frac{\operatorname{rp}^{+}\left(X_{1}, X_{2}\right)}{|G|^{\delta_{1}}}\right\}
$$

when $|G|$ is large enough in terms of $(\varepsilon, \delta, \gamma)$.

More precisely, one may take

$$
\delta_{1}=\frac{1}{2} \min \left(\frac{\delta \gamma}{2 c_{2}+1}, \frac{\varepsilon}{2 c_{2}}\right)
$$

where $c_{2}=973$ is as in Theorem 2.1 and

$$
C_{3} \leq 2^{14} C_{1} \leq 2^{2530}
$$

Proof. By definition, we have

$$
\operatorname{rp}\left(X_{1} X_{2}\right)=\sum_{g \in G} \mathbf{P}\left(X_{1} X_{2}=g\right)^{2}
$$

We now decompose the ranges of the distribution functions

$$
v_{i}(x)=\mathbf{P}\left(X_{i}=x\right)
$$

into dyadic intervals. Consider a parameter $I \geq 1$, to be chosen later, and decompose

$$
[\min \mathbf{P}(X=x), \max \mathbf{P}(X=x)] \subset[0,1]=\mathcal{I}_{0} \cup \mathcal{I}_{1} \cup \cdots \cup \mathcal{I}_{I}
$$

where

$$
\mathcal{I}_{i}= \begin{cases}] 2^{-i-1}, 2^{-i}\right] & \text { for } 0 \leq i<I, \\ {\left[0,2^{-I}\right]} & \text { for } i=I\end{cases}
$$


This gives two partitions of $G$ in subsets

$$
A_{j, i}=\left\{x \in G \mid v_{j}(x)=\mathbf{P}\left(X_{j}=x\right) \in \mathcal{I}_{i}\right\}
$$

for $j=1,2$. We note that

$$
\left|A_{j, i}\right| \leq 2^{i+1}
$$

for $j=1,2$ and $0 \leq i<I$.

Using this decomposition into the formula above, and the fact that

$$
\mathbf{P}\left(X_{1} X_{2}=g, X_{1} \in A_{1, I} \text { or } X_{2} \in A_{2, I}\right) \leq \mathbf{P}\left(X_{1} \in A_{1, I}\right)+\mathbf{P}\left(X_{2} \in A_{2, I}\right) \leq \frac{|G|}{2^{I-1}},
$$

we obtain

$$
\begin{aligned}
\operatorname{rp}\left(X_{1} X_{2}\right) & =\sum_{g \in G}\left(\sum_{0 \leq i, j \leq I} \mathbf{P}\left(X_{1} X_{2}=g, X_{1} \in A_{1, i}, X_{2} \in A_{2 . j}\right)\right)^{2} \\
& \leq 8|G|^{3} 2^{-2 I}+2 \sum_{g \in G}\left(\sum_{0 \leq i, j<I} \mathbf{P}\left(X_{1} X_{2}=g, X_{1} \in A_{1, i}, X_{2} \in A_{2, j}\right)\right)^{2} \\
& \leq 2^{3-2 I}|G|^{3}+2 I^{2} \sum_{0 \leq i, j<I} \sum_{g \in G} \mathbf{P}\left(X_{1} X_{2}=g, X_{1} \in A_{1, i}, X_{2} \in A_{2, j}\right)^{2}
\end{aligned}
$$

by the Cauchy-Schwarz inequality. Furthermore, the inner sum (say, $B\left(A_{1, i}, A_{2, j}\right)$ ) in the second term is given by

$$
\begin{aligned}
& B\left(A_{1, i}, A_{2, j}\right)=\sum_{g \in G} \mathbf{P}\left(X_{1} X_{2}=g, X_{1} \in A_{1, i}, \quad X_{2} \in A_{2, j}\right)^{2} \\
& =\sum_{g \in G}\left(\sum_{\substack{(x, y) \in A_{1, i} \times A_{2, j} \\
x y=g}} \mathbf{P}\left(X_{1}=x\right) \mathbf{P}\left(X_{2}=y\right)\right)^{2} \\
& =\sum_{\substack{x_{1}, X_{2} \in A_{1, i}, Y_{1}, Y_{2} \in A_{2, j} \\
X_{1} Y_{1}=X_{2} Y_{2}}} v_{1}\left(x_{1}\right) v_{1}\left(x_{2}\right) v_{2}\left(y_{1}\right) v_{2}\left(y_{2}\right) \\
& \leq 2^{-2 i-2 j}\left|\left\{\left(x_{1}, x_{2}, y_{1}, y_{2}\right) \in A_{1, i}^{2} \times A_{2, j}^{2} \mid x_{1} y_{1}=x_{2} y_{2}\right\}\right| \\
& =2^{-2 i-2 j} E\left(A_{1, i}, A_{2, j}\right),
\end{aligned}
$$

where $E(A, B)$ denotes the multiplicative energy. 
Thus, we have proved that

$$
\operatorname{rp}\left(X_{1} X_{2}\right) \leq 2^{3-2 I}|G|^{3}+2 I^{2} \sum_{0 \leq i, j<I} 2^{-2(i+j)} E\left(A_{1, i}, A_{2, j}\right)
$$

We now want to obtain upper-bounds in terms of the return probability $\mathrm{rp}^{+}\left(X_{1}, X_{2}\right)$. This is done in different ways, depending on the size of the subsets $A_{1, i}$ and $A_{2, j}$. We recall first the "trivial" bounds

$$
E(A, B) \leq \min \left(|A|^{2}|B|,|A||B|^{2}\right) .
$$

We claim that for all $i$ and $j$, we have

$$
2^{-2(i+j)} E\left(A_{1, i}, A_{2, j}\right) \leq 2^{4} \operatorname{rp}^{+}\left(X_{1}, X_{2}\right) e\left(A_{1, i}, A_{2, j}\right),
$$

and that, for all $\alpha \geq 1$, we have

$$
2^{-2(i+j)} E\left(A_{1, i}, A_{2, j}\right) \leq \alpha^{-1} \operatorname{rp}^{+}\left(X_{1}, X_{2}\right)
$$

unless

$$
\frac{\left|A_{1, i}\right|}{2^{i}} \geq \frac{1}{2 \sqrt{\alpha}}, \quad \frac{\left|A_{2, j}\right|}{2^{j}} \geq \frac{1}{2 \sqrt{\alpha}} .
$$

To see (4.8), we remark that

$$
\begin{aligned}
\operatorname{rp}^{+}\left(X_{1}, X_{2}\right) & \geq \frac{1}{2}\left(\operatorname{rp}\left(X_{1}\right)+\operatorname{rp}\left(X_{2}\right)\right)=\frac{1}{2} \sum_{g \in G}\left(\mathbf{P}\left(X_{1}=g\right)^{2}+\mathbf{P}\left(X_{2}=g\right)^{2}\right) \\
& \geq \frac{1}{2}\left(\frac{\left|A_{1, i}\right|}{2^{2+2 i}}+\frac{\left|A_{2, j}\right|}{2^{2+2 j}}\right) \geq \frac{1}{4} \frac{\left(\left|A_{1, i}\right|\left|A_{2, j}\right|\right)^{1 / 2}}{2^{i+j}} .
\end{aligned}
$$

for any choice of $i$ and $j$. Hence, we obtain

$$
\begin{aligned}
2^{-2(i+j)} E\left(A_{1, i}, A_{2, j}\right) & =2^{-2(i+j)} e\left(A_{1, i}, A_{2, j}\right)\left(\left|A_{1, i}\right|\left|A_{2, j}\right|\right)^{3 / 2} \\
& \leq 4 \operatorname{rp}^{+}\left(X_{1}, X_{2}\right) e\left(A_{1, i}, A_{2, j}\right) \frac{\left|A_{1, i}\right|\left|A_{2, j}\right|}{2^{i+j}} \\
& \leq \operatorname{16rp}^{+}\left(X_{1}, X_{2}\right) e\left(A_{1, i}, A_{2, j}\right)
\end{aligned}
$$

by (4.5). 
As for (4.9), if we assume that $2^{-2(i+j)} E\left(A_{1, i}, A_{2, j}\right)>\alpha^{-1} \mathrm{rp}^{+}\left(X_{1}, X_{2}\right)$, then we write simply

$$
2^{-2(i+j)}\left|A_{1, i}\right|^{2}\left|A_{2, j}\right| \geq 2^{-2(i+j)} E\left(A_{1, i}, A_{2, j}\right) \geq \alpha^{-1} \frac{\left|A_{2, j}\right|}{2^{2+2 j}},
$$

using (4.7), and obtain the first inequality of (4.10), the second being obtained symmetrically.

With these results, we now fix some parameter $\alpha \geq 1$, and let

$$
P_{\alpha}=\left\{(i, j)|0 \leq i, j<I, \quad| A_{1, i} \mid \geq 2^{i-1} \alpha^{-1} \quad \text { and } \quad\left|A_{2, j}\right| \geq 2^{j-1} \alpha^{-1}\right\}
$$

For $(i, j) \notin P_{\alpha}$, we have

$$
2^{-2(i+j)} E\left(A_{1, i}, A_{2, j}\right) \leq \alpha^{-2} \operatorname{rp}^{+}\left(X_{1}, X_{2}\right)
$$

by (4.9) and (4.10), and thus from (4.6), we have shown that

$$
\operatorname{rp}\left(X_{1} X_{2}\right) \leq 2^{3-2 I}|G|^{3}+2 \alpha^{-2} \operatorname{rp}^{+}\left(X_{1}, X_{2}\right) I^{4}+32 \operatorname{rp}^{+}\left(X_{1}, X_{2}\right) I^{2} \sum_{(i, j) \in P_{\alpha}} e\left(A_{1, i}, A_{2, j}\right)
$$

(estimating the size of the complement of $P_{\alpha}$ by $I^{2}$ ). We select

$$
I=\left\lceil\frac{2 \log 2|G|}{\log 2}\right\rceil \leq 3 \log (3|G|)
$$

and hence obtain

$$
\operatorname{rp}\left(X_{1} X_{2}\right) \leq \frac{1}{|G|}+2^{8} \operatorname{rp}^{+}\left(X_{1}, X_{2}\right)(\log 3|G|)^{2}\left\{\frac{(\log 3|G|)^{2}}{\alpha^{2}}+2 \sum_{(i, j) \in P_{\alpha}} e\left(A_{1, i}, A_{2, j}\right)\right\}
$$

We apply this bound with $\alpha=|G|^{\delta_{0}}$, where $\delta_{0}>0$ will be chosen later. Thus,

$$
\begin{aligned}
\operatorname{rp}\left(X_{1} X_{2}\right) \leq & \frac{1}{|G|}+2^{8} \operatorname{rp}^{+}\left(X_{1}, X_{2}\right)(\log 3|G|)^{4}|G|^{-2 \delta_{0}} \\
& +2^{9}(\log 3|G|)^{2} \operatorname{rp}^{+}\left(X_{1}, X_{2}\right) \sum_{(i, j) \in P_{\alpha}} e\left(A_{1, i}, A_{2, j}\right)
\end{aligned}
$$

Let then

$$
R_{\alpha}=\left\{(i, j) \in P_{\alpha} \mid e\left(A_{1, i}, A_{2, j}\right) \geq \alpha^{-1}\right\} \subset P_{\alpha}
$$


so that the contribution of those $(i, j) \in P_{\alpha}$ which are not in $R_{\alpha}$, together with the middle term, can be bounded by

$$
\frac{2^{13}(\log 3|G|)^{4}}{|G|^{\delta_{0}}} \operatorname{rp}^{+}\left(X_{1}, X_{2}\right) .
$$

We can now analyze the set $R_{\alpha}$; it turns out to be very restricted when $\delta_{0}$ is chosen small enough. By Theorem 2.1, for each $(i, j) \in R_{\alpha}$, there exists a $\beta_{1}$-approximate subgroup $\mathrm{H}_{i, j}$ and elements $\left(x_{i}, y_{j}\right) \in A_{1, i} \times A_{2, j}$ such that

$$
\left|\mathrm{H}_{i, j}\right| \leq \beta_{2}\left|A_{1, i}\right|, \quad\left|A_{1, i} \cap X_{i} \mathrm{H}_{i, j}\right| \geq \beta_{3}^{-1}\left|A_{1, i}\right|, \quad\left|A_{2, j} \cap \mathrm{H}_{i, j} Y_{j}\right| \geq \beta_{3}^{-1}\left|A_{2, j}\right|,
$$

and with tripling constant bounded by $\beta_{4}$, where the $\beta_{i}$ are bounded qualitatively by

$$
\beta_{i} \leq C_{1}|G|^{C_{2} \delta_{0}}
$$

for some absolute constants, which we take to be $c_{1}=2^{2516}, c_{2}=973$ using (2.2). We then note first that if $H_{i, j}$ denotes the "ordinary" subgroup generated by $\mathrm{H}_{i, j}$, we have

$$
\begin{aligned}
\mathbf{P}\left(X_{1} \in X_{i} H_{i, j}\right) & \geq \mathbf{P}\left(X_{1} \in x_{i} \mathrm{H}_{i, j}\right) \\
& \geq \mathbf{P}\left(X_{1} \in A_{1, i} \cap x_{i} \mathrm{H}_{i, j}\right) \\
& \geq \frac{1}{\beta_{3}} \frac{\left|A_{1, i}\right|}{2^{i+1}} \geq \frac{1}{4 \beta_{3} \alpha} \\
& \geq \frac{1}{4 c_{1}|G|^{\left(1+c_{2}\right) \delta_{0}}},
\end{aligned}
$$

where we used the definition of $P_{\alpha}$. If $\delta_{0}$ is small enough that

$$
\left(1+c_{2}\right) \delta_{0}<\gamma
$$

and if $|G|$ is large enough, this is not compatible with (4.2), and we can therefore assume that each $\mathrm{H}_{i, j}$ (if any!) generates the group $G$.

We next observe that $\mathrm{H}_{i, j}$ cannot be extremely small. Indeed, we have

$$
\left|\mathrm{H}_{i, j}\right| \geq\left|x_{i} \mathrm{H}_{i, j} \cap A_{1, i}\right| \geq \beta_{3}^{-1}\left|A_{1, i}\right|
$$


on the one hand, and by applying (4.2) with $H=1$, we can see that $A_{1, i}$ is not too small, namely

$$
\left|A_{1, i}\right| \geq \frac{\mathbf{P}\left(X_{1} \in A_{1, i}\right)}{\max _{g \in G} \mathbf{P}\left(X_{1}=g\right)} \geq|G|^{\gamma} \mathbf{P}\left(X_{1} \in A_{1, i}\right) \geq \frac{|G|^{\gamma}\left|A_{1, i}\right|}{2^{i+1}} \geq \frac{|G|^{\gamma}}{4 \alpha}
$$

using again the definition of $P_{\alpha}$.

This gives the lower bound

$$
\left|\mathrm{H}_{i, j}\right| \geq \frac{|G|^{\gamma}}{4 \alpha \beta_{3}} \geq \frac{1}{4 C_{1}}|G|^{\gamma_{1}}
$$

with $\gamma_{1}=\gamma-\delta_{0}\left(1+c_{2}\right)$ (which is $>0$ by (4.12)), and then leads to control of the tripling constant, namely

$$
\operatorname{trp}\left(\mathrm{H}_{i, j}\right) \leq \beta_{4} \leq C_{1}|G|^{C_{2} \delta_{0}} \leq C_{1}\left(4 C_{1}\right)^{2 \delta_{0} \gamma_{1}^{-1}}\left|\mathrm{H}_{i, j}\right|^{C_{2} \delta_{0} \gamma_{1}^{-1}}
$$

Since we assumed that $G$ is $\delta$-flourishing, we see from Definition 4.2 that if $\delta_{0}$ is such that

$$
\frac{c_{2} \delta_{0}}{\gamma_{1}}=\frac{c_{2} \delta_{0}}{\gamma-\left(1+c_{2}\right) \delta_{0}}<\delta
$$

and again if $|G|$ is large enough, the approximate subgroup $\mathrm{H}_{i, j}$ must in fact be very large, especially, it must satisfy

$$
\mathrm{H}_{i, j} \cdot \mathrm{H}_{i, j} \cdot \mathrm{H}_{i, j}=G
$$

and, in particular,

$$
\left|\mathrm{H}_{i, j}\right| \geq \frac{|G|}{\beta_{4}} \geq \frac{1}{C_{1}}|G|^{1-c_{2} \delta_{0}} .
$$

Intuitively, this implies that $X_{1}$ and $X_{2}$ are already rather uniformly distributed over $G$, and hence that $\operatorname{rp}^{+}\left(X_{1}, X_{2}\right)$ is already too small to be significantly improved at the level of $X_{1} X_{2}$. To express this idea concretely, we go back to the first stage of the argument, namely (4.6): the contribution to $\operatorname{rp}\left(X_{1} X_{2}\right)$ coming from $(i, j)$ was bounded by

$$
2^{-2(i+j)} E\left(A_{1, i}, A_{2, j}\right) \leq \frac{\left|A_{1, i}\right|\left|A_{2, j}\right|^{2}}{2^{2(i+j)}} \leq \frac{1}{2^{i-3}}
$$

by (4.5). But then we also have

$$
2^{i+1} \geq\left|A_{1, i}\right| \geq \frac{\left|\mathrm{H}_{i, j}\right|}{\beta_{2}} \geq \frac{|G|}{\beta_{2} \beta_{4}} \geq c_{1}^{-1}|G|^{1-c_{2} \delta_{0}}
$$


(observe that $\beta_{2} \beta_{4} \leq C_{1}|G|^{c_{2} \delta_{0}}$ ) and therefore

$$
2^{-2(i+j)} E\left(A_{1, i}, A_{2, j}\right) \leq 16 c_{1}|G|^{-1+2 c_{2} \delta_{0}} .
$$

Using again the trivial bound $I^{2} \leq 9(\log 3|G|)^{2}$ for the number of possible pairs $(i, j)$ to which this applies, the conclusion is an inequality

$$
\operatorname{rp}\left(X_{1} X_{2}\right) \leq \frac{1}{|G|}+2^{11} c_{1} \frac{(\log 3|G|)^{4}}{|G|^{1-c_{2} \delta_{0}}}+2^{13} \frac{(\log 3|G|)^{4}}{|G|^{\delta_{0}}} \operatorname{rp}^{+}\left(X_{1}, X_{2}\right)
$$

which holds (under the assumptions that $|G|$ is sufficiently large) for all $\delta_{0}$ small enough so that (4.12) and (4.15) are satisfied. It is elementary that (4.15) is stronger than (4.12) and is equivalent with

$$
\delta_{0}<\frac{\delta \gamma}{(1+\delta) c_{2}+\delta}
$$

which holds when $\delta_{0}<\delta \gamma /\left(2 c_{2}+1\right)$ (since we assume $\delta \leq 1$ ).

Thus, we can apply this for

$$
\delta_{0}=\min \left(\frac{\delta \gamma}{2 c_{2}+1}, \frac{\varepsilon}{2 c_{2}}\right)=2 \delta_{1},
$$

where $\delta_{1}$ is given by (4.4). Then for $|G|$ large enough, (4.16) implies (4.3), and hence we have finished the proof of Theorem 4.3.

We can summarize all this as follows (with the same remark as before concerning our handling of the conditions on the size of $G)$ :

Corollary 4.4 (The Bourgain-Gamburd expansion criterion). Let $\boldsymbol{c}=(c, d, \delta, \gamma)$ be a tuple of positive real numbers, and let $\mathcal{G}(\boldsymbol{c})$ be the family of all finite connected Cayley graphs $\mathcal{C}(G, S)$ for which the following conditions hold:

(1) We have $d(G) \geq|G|^{d}$;

(2) The group $G$ is $\delta$-flourishing;

(3) For the random walk ( $\left.X_{n}\right)$ on $G$ with $X_{0}=1$, we have that

$$
\mathbf{P}\left(X_{k} \in X H\right) \leq|G|^{-\gamma}
$$

for some $k \leq c \log |G|$ and all $x \in G$ and proper subgroups $H \subset G$. 
Then, for any $\Gamma \in \mathcal{G}(\boldsymbol{c})$ with $|\Gamma|$ large enough, the spectral gap of the normalized Laplace operator of $\Gamma$ satisfies

$$
\lambda_{1}(\Gamma) \geq 1-\exp \left(-\frac{d}{4 c j}\right)
$$

where

$$
j \leq 8 \max \left(\frac{2 c_{2}+1}{\delta \gamma}, \frac{16 c_{2}}{7 d}\right) .
$$

Note that it is not clear at this point that this corollary is not an empty statement (or one that applies at most to finitely many graphs with a bounded valency). But in the next section, we will check that it applies to the situation of Theorem 1.1 to prove that certain families of Cayley graphs are expanders.

Proof. Let $\Gamma=\mathcal{C}(G, S)$ be a graph in $\mathcal{G}(\boldsymbol{c})$. We will apply Theorem 4.3 with $\varepsilon=d / 2$ so that

$$
\delta_{1}=\frac{1}{2} \min \left(\frac{\delta \gamma}{2 c_{2}+1}, \frac{d}{4 c_{2}}\right) .
$$

When $|G|$ is large enough, we can rephrase the conclusion using the simpler inequality

$$
\operatorname{rp}\left(Y_{1} Y_{2}\right) \leq c_{3} \max \left(\frac{1}{|G|^{1-d / 2}}, \frac{\operatorname{rp}^{+}\left(Y_{1}, Y_{2}\right)}{|G|^{\delta_{1}}}\right) \leq \max \left(\frac{1}{|G|^{1-3 d / 4}}, \frac{\operatorname{rp}^{+}\left(Y_{1}, Y_{2}\right)}{|G|^{\delta_{1} / 2}}\right)
$$

for random variables $Y_{1}$ and $Y_{2}$ which satisfy the assumptions of this theorem.

Let $k=\lfloor c \log |G|\rfloor$ be given by (3). We apply the theorem to $Y_{1}=X_{2^{j} k}$ and $Y_{2}=$ $X_{2^{(j+1)} k} Y_{1}^{-1}$ for $j \geq 0$. These are indeed independent and symmetric random variables, and Conditions (2) and (3) imply that we can indeed apply Theorem 4.3 to these random variables for any $j \geq 2$. Since $Y_{1}$ and $Y_{2}$ are identically distributed, we have

$$
\operatorname{rp}^{+}\left(Y_{1}, Y_{2}\right)=\operatorname{rp}\left(Y_{1}\right)=\operatorname{rp}\left(X_{2^{j} k}\right)
$$

Thus, applying the theorem, we obtain by induction

$$
\operatorname{rp}\left(X_{2^{j} k}\right) \leq \operatorname{rp}\left(X_{k}\right)|G|^{-j \delta_{1} / 2} \leq|G|^{-j \delta_{1} / 2}
$$

when $j$ is such that

$$
|G|^{1-3 d / 4}>|G|^{j \delta_{1} / 2}
$$


and for larger $j$, we obtain

$$
\operatorname{rp}\left(X_{2^{j} k}\right) \leq|G|^{-1+3 d / 4} .
$$

In particular, we obtain this last inequality for

$$
j=\left\lceil\frac{2(1-3 d / 4)}{\delta_{1}}\right\rceil \leq \frac{4}{\delta_{1}} \leq 8 \max \left(\frac{2 c_{2}+1}{\delta \gamma}, \frac{4 c_{2}}{d}\right),
$$

which, by the "cycle-counting" inequality (4.1), gives

$$
\rho_{\Gamma} \leq\left(|G|^{1-d} \operatorname{rp}\left(X_{2^{j} k}\right)\right)^{1 /\left(2^{j} k\right)} \leq \exp \left(-\frac{d}{2^{j+3} C}\right),
$$

which thus proves the theorem.

\subsection{Expansion bounds for $\mathrm{SL}_{2}$}

Theorem 1.1 will now be proved by applying the criterion of Corollary 4.4. Thus, we will consider the groups $G_{p}=\mathrm{SL}_{2}\left(\mathbf{F}_{p}\right)$ for $p$ prime, for which Condition (1) of the BourgainGamburd criterion (which is purely a group-theoretic property) is given by

$$
d\left(\mathrm{SL}_{2}\left(\mathbf{F}_{p}\right)\right)=\frac{p-1}{2}
$$

for $p \geq 3$ (a result of Frobenius), which gives a value of $d$ arbitrarily close to $\frac{1}{3}$, for $p$ large enough. Condition (2) is given by Helfgott's Theorem, with $\delta=\frac{1}{3024}$. Note that it is purely a property of the groups $\mathrm{SL}_{2}\left(\mathbf{F}_{p}\right)$.

Condition (3), on the other hand, depends on the choice of generating sets. The symmetric generating sets $S_{p}$ in Theorem 1.1 are assumed to be obtained by reduction modulo $p$ of a fixed symmetric subset $S \subset \mathrm{SL}_{2}(\mathbf{Z})$. We will argue first under the additional assumption that $S \subset \mathrm{SL}_{2}(\mathbf{Z})$ generates a free group.

We begin with a classical proposition, the idea of which goes back to Margulis. For the statement, recall that the norm of a matrix $g \in G L_{n}(\mathbf{C})$ is defined by

$$
\|g\|=\max _{v, w \neq 0} \frac{|\langle g v, w\rangle|}{\|v\|\|w\|}
$$

where $\langle\cdot, \cdot\rangle$ is the standard inner product on $\mathbf{C}^{n}$. This satisfies

$$
\left\|g_{1} g_{2}\right\| \leq\left\|g_{1}\right\|\left\|g_{2}\right\|, \quad \max _{i, j}\left|g_{i, j}\right| \leq\|g\| \quad \text { for } g=\left(g_{i, j}\right)
$$

the latter because $g_{i, j}=\left\langle g e_{i}, e_{j}\right\rangle$ in terms of the canonical basis. 
Proposition 4.5 (Large girth for finite Cayley graphs). Let $S \subset \mathrm{SL}_{2}$ (Z) be a symmetric set, and let $\Gamma=\mathcal{C}(G, S)$ be the corresponding Cayley graphs. Let $\tau>0$ be defined by

$$
\tau^{-1}=\log \max _{s \in S}\|s\|>0
$$

which depends only on $S$.

(1) For all primes $p$ and all $r<\tau \log (p / 2)$, where $G_{p}=\mathrm{SL}_{2}\left(\mathbf{F}_{p}\right)$, the subgraph $\Gamma_{r}$ induced by the ball of radius $r$ in $\Gamma$ maps injectively to $\mathcal{C}\left(G_{p}, S\right)$.

(2) If $G$ is freely generated by $S$, in particular, $1 \notin S$, the Cayley graph $\mathcal{C}\left(G_{p}, S\right)$ contains no cycle of length $<2 \tau \log (p / 2)$, that is, its $\operatorname{girth} \operatorname{girth}\left(\mathcal{C}\left(\mathrm{G}_{\mathrm{p}}, \mathrm{S}\right)\right)$ is at least $2 \tau \log (p / 2)$.

Proof. The main point is that if all coordinates of two matrices $g_{1}, g_{2} \in \mathrm{SL}_{2}(\mathbf{Z})$ are less than $p / 2$ in absolute value, a congruence $g_{1} \equiv g_{2}(\bmod p)$ is equivalent to the equality $g_{1}=g_{2}$. And because $G$ is freely generated by $S$, knowing a matrix in $G$ is equivalent to knowing its expression as a word in the generators in $S$.

Thus, let $x$ be an element in the ball of radius $r$ centered at the origin. By definition, $x$ can be expressed as

$$
X=s_{1} \cdots s_{m}
$$

with $m \leq r$ and $s_{i} \in S$. Using (4.18), we obtain

$$
\max _{i, j}\left|x_{i, j}\right| \leq\|x\| \leq\left\|s_{1}\right\| \cdots\left\|s_{m}\right\| \leq \mathrm{e}^{m / \tau} \leq \mathrm{e}^{r / \tau}
$$

Applying the beginning remark and this fact to two elements $x$ and $y$ in the ball $\mathcal{B}_{1}(r)$ of radius $r$ centered at 1 , for $r$ such that $\mathrm{e}^{r / \tau}<\frac{p}{2}$, it follows that $x \equiv y(\bmod p)$ implies $x=y$, which is (1).

Then (2) follows because any embedding of a cycle $\gamma: C_{m} \rightarrow \mathcal{C}\left(G_{p}, S\right)$ such that $\gamma(0)=1$ and such that

$$
d(1, \gamma(i)) \leq m / 2<\tau \log (p / 2)
$$

for all $i$ can be lifted to the cycle (of the same length) with image in the Cayley graph of $G$ with respect to $S$, and if $S$ generates freely $G$, the latter graph is a tree. Thus, a cycle of length $m=\operatorname{girth}\left(\mathcal{C}\left(\mathrm{G}_{\mathrm{p}}, \mathrm{S}\right)\right)$ must satisfy $m / 2 \geq \tau \log (p / 2)$. 
We can now check Condition (3) in the Bourgain-Gamburd criterion, first for cosets of the trivial subgroup, that is, for the probability that $X_{n}$ be a fixed element when $n$ is of size $c \log p$ for some fixed (but small) $c>0$. As we did earlier, we clearly mark where we impose conditions on the size of $p$, and these will be made explicit in Section 4.3.

Corollary 4.6 (Decay of probabilities). Let $S \subset \mathrm{SL}_{2}(\mathrm{Z})$ be a symmetric set, $G$ the subgroup generated by $S$. Assume that $S$ freely generates $G$. Let $p$ be a prime such that the reduction $S_{p}$ of $S$ modulo $p$ generates $G_{p}=\mathrm{SL}_{2}\left(\mathbf{F}_{p}\right)$, and let $\left(X_{n}\right)$ be the random walk on $\mathcal{C}\left(G_{p}, S_{p}\right)$ with $X_{0}=1$. Let

$$
\tau^{-1}=\log \max _{s \in S}\|s\|>0
$$

as in Proposition 4.5.

Fix a constant $c$ with $0<c \leq 1$. If $p$ is large enough, depending on $c$ and $S$, then for

$$
n=c\lfloor\tau \log (p / 2)\rfloor
$$

and any $x \in \mathrm{SL}_{2}\left(\mathbf{F}_{p}\right)$, we have

$$
\mathbf{P}\left(X_{n}=x\right) \leq\left|G_{p}\right|^{-c \gamma_{1}}
$$

where

$$
\gamma_{1}=\frac{\tau\left(\log \left(\frac{2}{\sqrt{3}} \sqrt{|S|}\right)\right)}{8} .
$$

More precisely, this holds for all

$$
p \geq \max \left(17,2 \exp \left(\frac{2}{c \tau}\right)\right) .
$$

The "extra" parameter $c$ will be useful in the argument involving all proper subgroups $H$ below.

Proof. There exists $\tilde{X} \in G$ such that $\tilde{X}$ reduces to $x$ modulo $p$ and $\tilde{X}$ is at the same distance to 1 as $x$, and by Proposition 4.5, (2), we have

$$
\mathbf{P}\left(X_{n}=x\right)=\mathbf{P}\left(\tilde{X}_{n}=\tilde{X}\right)
$$


for $n \leq \tau \log (p / 2)$, where $\left(\tilde{X}_{n}\right)$ is the random walk starting at 1 on the $|S|$-regular tree $\mathcal{C}(G, S)$. By a well-known result of Kesten [14], we have

$$
\mathbf{P}\left(\tilde{X}_{n}=\tilde{X}\right) \leq r^{-n} \quad \text { with } r=\frac{|S|}{2 \sqrt{|S|-1}},
$$

for all $n \geq 1$ and all $\tilde{x} \in G$. Since $c \leq 1$, we have

$$
n=c\lfloor\tau \log (p / 2)\rfloor \geq c \tau \log (p / 2)-1,
$$

and we obtain

$$
\mathbf{P}\left(X_{n}=x\right) \leq r\left(\frac{p}{2}\right)^{-c \tau \log r} \leq\left(\frac{p}{2}\right)^{-\frac{1}{2} c \tau \log r},
$$

for $p \geq 2 r^{2 /(c \tau \log r)}$. Using the inequality

$$
\frac{p}{2} \geq\left|G_{p}\right|^{1 / 4}
$$

for $p \geq 17$, this becomes

$$
\mathbf{P}\left(X_{n}=x\right) \leq\left|G_{p}\right|^{-c \tau(\log r) / 8}
$$

for all $p \geq \max \left(17,2 r^{2 /(c \tau \log r)}\right)$. Since $r \geq \frac{2}{\sqrt{3}} \sqrt{|S|}$, we obtain the desired result.

In order to deal with cosets of other proper subgroups of $\mathrm{SL}_{2}\left(\mathbf{F}_{p}\right)$, we will exploit the fact that those subgroups are very well understood, and in particular, there is no proper subgroup that is "both big and complicated". Precisely, by results going back to Dickson (see, e.g., the account in [23, Chapter 6] for $\mathrm{PSL}_{2}\left(\mathbf{F}_{p}\right)$, from which the result for $\mathrm{SL}_{2}\left(\mathbf{F}_{p}\right)$ follows easily), one knows that for $p \geq 5$, if $H \subset \mathrm{SL}_{2}\left(\mathbf{F}_{p}\right)$ is a proper subgroup, one of the following two properties holds:

(1) The order of $H$ is at most 120;

(2) For all $\left(x_{1}, x_{2}, x_{3}, x_{4}\right) \in H$, we have

$$
\left[\left[x_{1}, x_{2}\right],\left[x_{3}, x_{4}\right]\right]=1 .
$$

The first ones are "small", and will be easy to handle using (4.20). The second are, from the group-theoretic point of view, not very complicated (their commutator subgroups are abelian). The following ad hoc lemma takes care of them (note that this 
is the only place where using prime fields $\mathbf{F}_{p}$ instead of arbitrary finite fields really simplifies the argument, since (4.23) does not hold for proper subgroups of, say, $\left.\mathrm{SL}_{2}\left(\mathrm{~F}_{p^{2}}\right)\right)$ :

Proposition 4.7. Let $k \geq 2$ be an integer and let $W \subset F_{k}$ be a subset of the free group on $k$ generators $\left(a_{1}, \ldots, a_{k}\right)$ such that

$$
\left[\left[x_{1}, x_{2}\right],\left[x_{3}, x_{4}\right]\right]=1
$$

for all $\left(x_{1}, x_{2}, x_{3}, x_{4}\right) \in W$. Then for any $m \geq 1$, we have

$$
\left|\left\{x \in W \mid d_{T}(1, x) \leq m\right\}\right| \leq(4 m+1)(8 m+1) \leq 45 m^{2}
$$

where $T$ is the $|S|$-regular tree $\mathcal{C}\left(F_{k}, S\right), S=\left\{a_{i}^{ \pm 1}\right\}$.

Proof. The basic fact we need is that the condition $[x, y]=1$ is very restrictive in $F_{k}$ : precisely, for a fixed $x \neq 1$, we have $[x, y]=1$ if and only if $y \in C_{F_{k}}(x)$, which is an infinite cyclic group. Denoting a generator by $z$, we find

$$
\left|\left\{y \in \mathcal{B}_{1}(m) \mid[x, y]=1\right\}\right|=\left|\left\{h \in \mathbf{Z} \mid d_{T_{k}}\left(1, z^{h}\right) \leq m\right\}\right| \leq 2 m+1
$$

since (a standard fact in free groups) we have $d_{T}\left(1, z^{h}\right) \geq|h|$.

Let $W$ be a set satisfying the assumption (4.24), which we assume to be not reduced to $\{1\}$. We denote $W_{m}=W \cap \mathcal{B}_{1}(m)$. First, if $[x, y]=1$ for all $x, y \in W_{m}$, then by taking a fixed $x \neq 1$ in $W_{m}$, we obtain $W_{m} \subset C_{F_{k}}(x) \cap \mathcal{B}_{1}(m)$, and (4.25) gives the result.

Otherwise, fix $x_{0}$ and $y_{0}$ in $W_{m}$ such that $a=\left[x_{0}, y_{0}\right] \neq 1$. Then, for all $y$ in $W_{m}$ we have $\left[a,\left[x_{0}, y\right]\right]=1$. Noting that $d_{T}\left(1,\left[x_{0}, y\right]\right) \leq 4 m$, it follows again from the above that the number of possible values of $\left[x_{0}, y\right]$ is at most $8 m+1$ for $y \in W_{m}$.

Now for one such value $b=\left[x_{0}, y\right]$, we consider how many $y_{1} \in W_{m}$ may satisfy $\left[x_{0}, y_{1}\right]=b$. We have $\left[x_{0}, y\right]=\left[x_{0}, y_{1}\right]$ if and only if $\varphi\left(y^{-1} y_{1}\right)=y^{-1} y_{1}$, where $\varphi(y)=x_{0} y x_{0}^{-1}$ denotes the inner automorphism of conjugation by $x_{0}$. Hence, $y_{1}$ satisfies $\left[x_{0}, y_{1}\right]=b$ if and only if $\varphi\left(y^{-1} y_{1}\right)=y^{-1} y_{1}$, which is equivalent to $y^{-1} y_{1} \in C_{F_{k}}\left(x_{0}\right)$. Since $y^{-1} y_{1}$ is an element at distance $\leq 2 m$ of 1 if $y$ and $y_{1}$ are in $\mathcal{B}_{1}(m)$, applying (4.25) gives

$$
\left|\left\{y_{1} \in \mathcal{B}_{1}(m) \mid\left[x_{0}, y_{1}\right]=\left[x_{0}, y\right]\right\}\right| \leq 4 m+1,
$$

and hence we have $\left|W_{m}\right| \leq(4 m+1)(8 m+1)$ in that case, which proves the result. 
Using Corollary 4.6, we finally verify fully Condition (3) in Corollary 4.4:

Corollary 4.8 (Decay of probabilities, II). Let $S \subset \mathrm{SL}_{2}(\mathrm{Z})$ be a symmetric set, $G$ the subgroup generated by $S$. Assume that $S$ freely generates $G$. Let $p$ be a prime such that the reduction $S_{p}$ of $S$ modulo $p$ generates $G_{p}=\mathrm{SL}_{2}\left(\mathbf{F}_{p}\right)$, and let $\left(X_{n}\right)$ be the random walk on $\mathcal{C}\left(G_{p}, S_{p}\right)$ with $X_{0}=1$. Let

$$
\tau^{-1}=\log \max _{s \in S}\|s\|>0
$$

as in Proposition 4.5.

If $p$ is large enough, then for

$$
n=\left\lfloor\frac{\tau}{32} \log (p / 2)\right\rfloor
$$

any $x \in \mathrm{SL}_{2}\left(\mathbf{F}_{p}\right)$ and any proper subgroup $H \subset \mathrm{SL}_{2}\left(\mathbf{F}_{p}\right)$, we have

$$
\mathbf{P}\left(X_{n} \in X H\right) \leq\left|G_{p}\right|^{-\gamma}
$$

where

$$
\gamma=\frac{\tau\left(\log \left(\frac{2}{\sqrt{3}} \sqrt{|S|}\right)\right)}{2^{9}} .
$$

Proof. We start by noting that

$$
\mathbf{P}\left(X_{n} \in X H\right)^{2} \leq \mathbf{P}\left(X_{2 n} \in H\right)
$$

for all $x \in G_{p}$ and all subgroups $H \subset G_{p}$.

Consider first the case where (4.23) holds for $H$. Let $\tilde{H} \subset G$ be the pre-image of $H$ under reduction modulo $p$. If $2 n \leq \tau \log (p / 2)$, then as in the proof of Corollary 4.6, we obtain

$$
\mathbf{P}\left(X_{2 n} \in H\right)=\mathbf{P}\left(\tilde{X}_{2 n} \in \tilde{H}\right)
$$

Provided $n$ also satisfies the stronger condition $n \leq m=\frac{1}{16} \tau \log (p / 2)$, any commutator

$$
\left[\left[x_{1}, x_{2}\right],\left[x_{3}, x_{4}\right]\right]
$$

with $x_{i} \in \tilde{H} \cap \mathcal{B}_{1}(n)$ is an element at distance at most $\tau \log (p / 2)$ from 1 in the tree $\mathcal{C}(G, S)$, which reduces to the identity modulo $p$ by (4.23), and therefore must be itself equal 
to 1 . In other words, we can apply Proposition 4.7 to $W=\tilde{H} \cap \mathcal{B}_{1}(m)$ to deduce the upper bound

$$
\left|\tilde{H} \cap \mathcal{B}_{1}(m)\right| \leq 45 m^{2} .
$$

We now take

$$
n=\frac{1}{32}\lfloor\tau \log (p / 2)\rfloor,
$$

and we derive

$$
\mathbf{P}\left(X_{2 n} \in H\right) \leq\left|\tilde{H} \cap \mathcal{B}_{1}(m)\right|\left(\max _{x \in G_{p}} \mathbf{P}\left(X_{2 n}=x\right)\right) \leq 45 m^{2}\left|G_{p}\right|^{-\gamma_{1} / 16}
$$

(where $\gamma_{1}$ is given by (4.21), as in Corollary 4.6), and hence

$$
\mathbf{P}\left(X_{n} \in X H\right) \leq \frac{\sqrt{45}}{16} \tau(\log p / 2)\left|G_{p}\right|^{-\gamma_{1} / 32} \leq\left|G_{p}\right|^{-\gamma_{1} / 64}
$$

provided $p$ is large enough, which is the conclusion in that case.

On the other hand, if (4.23) does not hold, we have $|H| \leq 120$, and for the same value of $n$ we obtain

$$
\mathbf{P}\left(X_{n} \in X H\right) \leq 120\left|G_{p}\right|^{-\gamma_{1} / 32} \leq\left|G_{p}\right|^{-\gamma_{1} / 64}
$$

for $p$ large enough, by Corollary 4.6 with $c=\frac{1}{32}$. This gives again the desired result.

The following upper-bound on $\gamma$ was suggested by the referee:

Lemma 4.9. With notation as in Corollary 4.8, we have

$$
\gamma \leq 2^{-5}
$$

Proof. For $n \geq 1$, the cardinality of the ball $\mathcal{B}_{1}(n)$ is at least $(|S|-1)^{n}$, and is at most

$$
\mid\left\{g \in M_{2}(\mathrm{Z})|| g_{i, j} \mid \leq\left(\max _{s \in S}\|s\|\right)^{n} \text { for } 1 \leq i, j \leq 2\right\}
$$

by (4.18). Thus, denoting $\Delta=\max _{s \in S}\|s\|$, we find

$$
\log (|S|-1) \leq 4 \log (2 \Delta+1)
$$


and hence

$$
\gamma=2^{-9} \frac{\log \left(\frac{2}{\sqrt{3}} \sqrt{|S|}\right)}{\log (\Delta)} \leq 2^{-7} \frac{\log (2 \Delta+1)}{\log (\Delta)}
$$

Now we note that either $\Delta \geq 2$, or $S$ is contained in the finite set of matrices in $\mathrm{SL}_{2}(\mathbf{Z})$ where all coefficients are in $\{-1,0,1\}$. There are 20 such matrices, and all those which are not of finite order are parabolic. For these, we have $\|s\| \geq \sqrt{2}$, and therefore $\Delta \geq \sqrt{2}$ in all cases, and hence

$$
\gamma \leq 2^{-7} \frac{\log (2 \sqrt{2}+1)}{\log (\sqrt{2})} \leq 2^{-5}
$$

\subsection{Summary}

We can now summarize how to obtain an explicit spectral gap, for large enough $p$, in the situation of Theorem 1.1, finishing the proof. We then explain how to quantify the condition on $p$.

We first consider the case where $S \subset \mathrm{SL}_{2}(\mathbf{Z})$ freely generates a free group of rank $\geq 2$ (in which case it is automatically Zariski-dense in $\mathrm{SL}_{2}$ ).

Step 1 (when p is large enough). We have

$$
d\left(G_{p}\right)=\frac{p-1}{2}
$$

for $p \geq 3$. In particular, $d\left(G_{p}\right) \geq\left|G_{p}\right|^{d}$ for any $d<\frac{1}{3}$ provided $p$ is large enough in terms of $d$. Moreover, by Theorem 1.2, those groups are $\delta$-flourishing with $\delta=\frac{1}{3024}$.

For the random walk $\left(X_{n}\right)$ on $G_{p}$ associated to the generating set $S_{p}$, with $X_{0}=1$, we have

$$
\mathbf{P}\left(X_{k} \in X H\right) \leq|G|^{-\gamma}
$$

when

$$
k=\left\lfloor\frac{\tau}{32} \log \left(\frac{p}{2}\right)\right\rfloor \leq \frac{\tau}{96} \log \left(\left|G_{p}\right|\right)
$$

with

$$
\tau^{-1}=\log \max _{s \in S}\|s\|, \quad \gamma=\frac{\tau \log \left(\frac{2}{\sqrt{3}} \sqrt{|S|}\right)}{2^{9}} .
$$


by (4.19) and (4.27). Thus, in Corollary 4.4, we can take $c=\frac{1}{96}$. The number of times we apply the basic $L^{2}$-flattening inequality is bounded by

$$
j \leq 8 \max \left(\frac{2 c_{2}+1}{\delta \gamma}, \frac{4 c_{2}}{d}\right) \leq 8 \max \left(\frac{1947 \cdot 3024}{\gamma}, 15000\right)=47101824 \gamma^{-1} \leq 2^{26} \gamma^{-1}
$$

(using Lemma 4.9) and the spectral gap satisfies

$$
\lambda_{1}(\Gamma) \geq 1-\exp \left(-\frac{d}{2^{j+3} C}\right)=1-\exp \left(-\frac{d}{2^{j+3} C}\right) \geq \frac{d}{2^{j+4} C},
$$

for all $p$ large enough. For $p \geq 17$, we take $d=\frac{1}{4}$, and this gives

$$
\lambda_{1}(\Gamma) \geq \frac{d}{2^{j+4} C} \geq \frac{3}{2^{j+1}} \geq 2^{-2^{26} \gamma^{-1}} .
$$

Except that we incorporated the factor $2^{9}$ from the current value of $\gamma$ to the constant factor (for esthetic reasons), this gives (1.1).

Step 2 (how large is "large enough"). We gather here, as a series of inequalities to be satisfied by $p$, the conditions under which we can apply the previous lower bound. These we gather from the proofs of the results of this section. First come inequalities that make explicit the condition that $|G|$ be large enough in Theorem 4.3, which are easily translated into conditions on $p$ since $\left|\mathrm{SL}_{2}\left(\mathbf{F}_{p}\right)\right|=p\left(p^{2}-1\right)$.

- In order that (4.11) contradict (4.2), we must have

$$
|G|^{\gamma-\delta_{0}\left(1+c_{2}\right)}>4 C_{1}
$$

- In order that (4.14) contradicts the growth alternative of Helfgott's Theorem, it is enough that

$$
|G|^{\gamma_{1}}>4 c_{1}\left\{C_{1}\left(4 c_{1}\right)^{\gamma_{1}^{-1}}\right\}^{\left(\delta-c_{2} \delta_{0} \gamma_{1}^{-1}\right)^{-1}}
$$

where $\gamma_{1}=\gamma-\left(1+c_{2}\right) \delta_{0}$ (in view of (4.13)). (This is not the same $\gamma_{1}$ that occurs in the proof of the decay of probabilities.)

- In order that (4.16) give (4.3) when $\delta_{1}$ satisfies (4.4), it is enough that

$$
|G|^{\varepsilon-2 c_{2} \delta_{0}} \geq(\log 3|G|)^{4},
$$


and that

$$
|G|^{\delta_{0}} \geq c_{1}^{-2}(\log 3|G|)^{4}
$$

- In order that (4.17) hold, we must have

$$
\min \left(|G|^{d / 4},|G|^{\delta_{1} / 2}\right) \geq c_{3} .
$$

Now we list the conditions needed to apply the Bourgain-Gamburd criterion in the situation of Theorem 1.1, when $S$ freely generates a free group of rank $|S| / 2 \geq 2$.

- We need

$$
p \geq \max \left(17,2 \exp \left(\frac{2}{c \tau}\right)\right)
$$

by (4.22).

- In order that the last inequality in (4.28) hold, as well as (4.29), it is enough that

$$
\left|\mathrm{SL}_{2}\left(\mathbf{F}_{p}\right)\right|^{\gamma} \geq \max \left(120,\left(\log \frac{p}{2}\right)\right) .
$$

Remark 4.10. Below in Section 4.5 is found a straightforward PARI/GP [20] that computes the lower-bound of Step 1 for the spectral gap, given the set of matrices $S$, and that can also be used to determine for which $p$ the bound is known to be applicable.

We finally explain how to reduce the full statement of Theorem 1.1 to the case where the given symmetric subset $S \subset \mathrm{SL}_{2}(\mathbf{Z})$ generates a free group, which is the one treated by the Bourgain-Gamburd method.

For a given $S \subset \mathrm{SL}_{2}(\mathbf{Z})$ which generates a Zariski-dense subgroup $G$ of $\mathrm{SL}_{2}$, the intersection $G \cap \Gamma(2)$, where $\Gamma(2)$ is the principal congruence subgroup modulo 2, is a free subgroup of finite index in $G$. From a free generating set, one can extract two generators $s_{1}, s_{2} \in G$ to obtain a free subgroup of rank 2 of $G$, say $G_{1}$ (since $G \cap \Gamma(2)$ has finite index in $G$, it is still Zariski-dense, and hence has rank at least 2). This subgroup is still Zariski-dense. We can then compare the expansion for the Cayley graphs of $\mathrm{SL}_{2}\left(\mathbf{F}_{p}\right)$ with respect to $S$ and to $S_{1}=\left\{s_{1}^{ \pm 1}, s_{2}^{ \pm 1}\right\}$.

For $p$ large enough so that $G_{p}=\mathrm{SL}_{2}\left(\mathbf{F}_{p}\right)$ is generated both by $S$ modulo $p$ and $S_{1}$ modulo $p$, we have

$$
d(x, y) \leq C d_{1}(x, y),
$$


where $d_{1}(\cdot, \cdot)$ is the distance in the Cayley graph $\Gamma_{1}=\mathcal{C}\left(G_{p}, S_{1}(\bmod p)\right)$, and $d(\cdot, \cdot)$ the distance in $\Gamma_{2}=\mathcal{C}\left(G_{p}, S(\bmod p)\right)$ and $C$ is the maximum of the word length of $s_{1}$ and $s_{2}$ with respect to $S$. Hence, by a standard lemma (see, e.g., [16, Lemma 3.1.16], applied to $\Gamma_{1}$ and $\Gamma_{2}$ with $f$ the identity), the expansion constants satisfy

$$
h\left(\mathcal{C}\left(G_{p}, S(\bmod p)\right)\right)=h\left(\Gamma_{2}\right) \geq w^{-1} h\left(\mathcal{C}\left(G_{p}, S_{1}(\bmod p)\right)\right)
$$

with

$$
w=4 \sum_{j=1}^{\lfloor C\rfloor}|S|^{j-1} .
$$

In particular, using Theorem 1.1 for $G_{1}$, we obtain the expansion property for $G$, and we can bound the spectral gap explicitly once we know expressions for the generators $s_{1}$ and $s_{2}$ in terms of those in $S$.

As the referee pointed out, Breuillard and Gelander [3, Theorem 1.2] have proved a strong uniform version of the Tits alternative which implies that there exists an absolute constant $N \geq 1$ such that, for any Zariski-dense subgroup $G \subset \mathrm{SL}_{2}$ (Z), and for any symmetric generating set $S \subset G$, the combinatorial ball of radius $N$ in $\mathcal{C}(G, S)$ contains two elements which generate a free subgroup of rank 2 of $G$. If a concrete value of $N$ was known (which does not seem to be the case yet), one could use the above argument to state a version of the second part of Theorem 1.1 without the assumption of freeness.

\subsection{Diameter bound}

Now, we can also prove quickly Corollary 1.4. Let $S_{1}=S \cup\{1\}$. By Proposition 4.5, if we let

$$
r=\left\lfloor\tau \log \frac{p}{2}\right\rfloor
$$

where $\tau$ is defined by (4.19), the size of $S_{1}{ }^{(r)}$ is at least the size of a ball of radius $r$ in a $|S|$-regular tree, which is well known to be at least $s^{r}$, where $s=|S|-1$.

For $p \geq 17$, this gives

$$
S_{1}{ }^{(r)} \geq s^{-1}\left(\frac{p}{2}\right)^{r} \geq s^{-1}\left|\mathrm{SL}_{2}\left(\mathbf{F}_{p}\right)\right|^{\tau(\log r) / 4},
$$

and if $p \geq \exp \left(2 \tau^{-1}\right)$, this becomes

$$
S_{1}^{(r)} \geq\left|\mathrm{SL}_{2}\left(\mathbf{F}_{p}\right)\right|^{\delta_{2}},
$$


where

$$
\delta_{2}=\frac{\tau(\log s)}{8}>0
$$

Now we apply repeatedly Helfgott's Theorem with $H=S_{1}{ }^{(r)}$. For $j$ such that

$$
j \geq \frac{\log \left(\delta_{2}^{-1}\right)}{\log (1+\delta)},
$$

the $3^{j}$-fold product of $H$ must be equal to $\mathrm{SL}_{2}\left(\mathbf{F}_{p}\right)$, and hence we obtain

$$
\operatorname{diam} \mathcal{C}\left(\mathrm{SL}_{2}\left(\mathbf{F}_{p}\right), S\right) \leq 3^{j} r \leq 3^{j-1}\left(\log \left|\mathrm{SL}_{2}\left(\mathbf{F}_{p}\right)\right|\right)
$$

and taking

$$
j=\left\lceil\frac{\log \left(\delta_{2}^{-1}\right)}{\log (1+\delta)}\right\rceil,
$$

this gives the bound

$$
\operatorname{diam} \mathcal{C}\left(\mathrm{SL}_{2}\left(\mathbf{F}_{p}\right), S\right) \leq 3^{\log \left(\delta_{2}^{-1}\right) / \log (1+\delta)}\left(\log \left|\mathrm{SL}_{2}\left(\mathbf{F}_{p}\right)\right|\right)
$$

\subsection{Script}

Here is a PARI/GP [20] script that performs the computations needed to obtain an explicit spectral for Theorem 1.1, given as input a set of matrices $S$ which generate a free group (this condition is not checked).

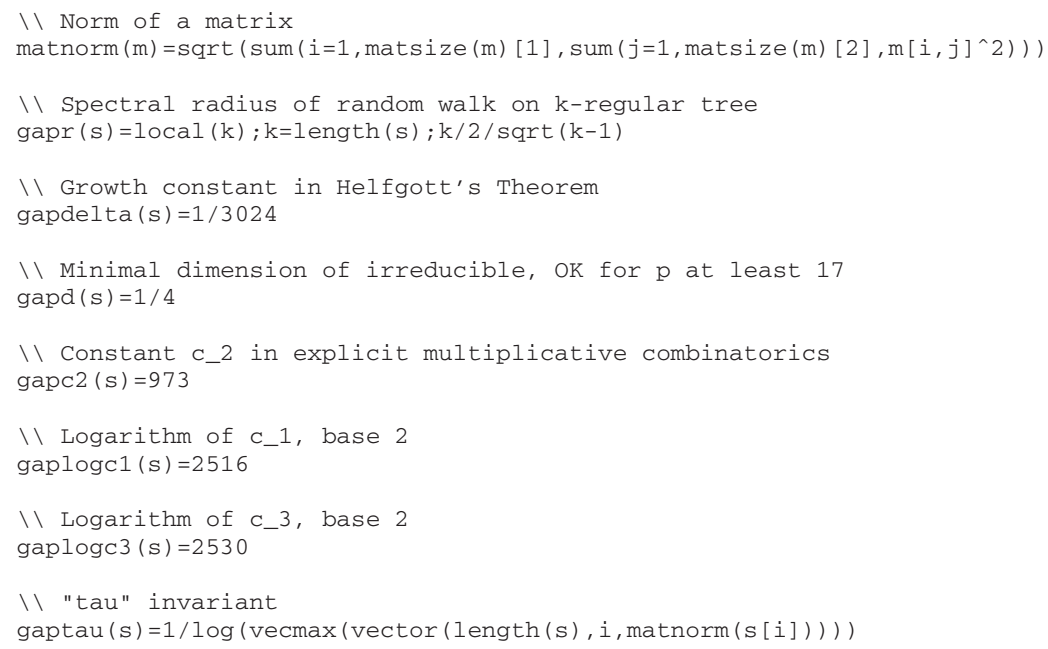




\section{E. Kowalski}

\\Value of gamma for $p$ large enough

gapgamma $(\mathrm{s})=\operatorname{gaptau}(\mathrm{s}) * \log (2 / \operatorname{sqrt}(3) * \operatorname{sqrt}(\operatorname{length}(\mathrm{s}))) / 2^{\wedge} 9$

$\backslash \backslash$ Bound for minus the logarithm in base 2 of spectral gap

$\backslash \backslash$ for $p$ large enough

gaploggap $(s)=2^{\wedge} 26 /$ gapgamma $(s)$

\\Value of delta_0

gapdelta0 $(\mathrm{s})=\min (\operatorname{gapdelta}(\mathrm{s}) * \operatorname{gapgamma}(\mathrm{s}) /(2 * \operatorname{gapc} 2(\mathrm{~s})+1), \operatorname{gapd}(\mathrm{s}) / 8 / \operatorname{gapc} 2(\mathrm{~s}))$

$\backslash \backslash$ Value of delta_1

gapdelta1 $(\mathrm{s})=1 / 2 * \min (\operatorname{gapdelta}(\mathrm{s}) * \operatorname{gapgamma}(\mathrm{s}) /(2 * \operatorname{gapc} 2(\mathrm{~s})+1), \operatorname{gapd}(\mathrm{s}) / 8 / \operatorname{gapc} 2(\mathrm{~s}))$

$\backslash$ Value of gammal in lower-bound conditions

gapgamma1 $(\mathrm{s})$ =gapgamma $(\mathrm{s})$-gapdelta1 $(\mathrm{s}) *(1+\operatorname{gapc} 2(\mathrm{~s}))$

$\backslash \backslash$ First minimal value on $\log p$, base 2

gaplogmin1 $(\mathrm{s})=(2+\operatorname{gaplogc1}(\mathrm{s})) / 3 /(\operatorname{gapgamma}(\mathrm{s})-\operatorname{gapdelta1}(\mathrm{s}) *(1+\operatorname{gapc} 2(\mathrm{~s})))$

\\Second minimal value on $\log p$, base 2

gaplogmin2 (s) =1/3/gapgamma1 (s) * (2+gaplogc1 (s) +1/(gapdelta (s)-gapc2 (s) *gapdelta0 (s)/gapgamma1 (s)) * (gaplogc1 (s) +1/gapgamma1 (s)*(2+gaplogc1 (s))))

$\backslash \backslash$ Is $\log (p)=l p$ larger than third minimal value on $\log p$ (base e)?

gapislogmin $3(s, 1 p)=$ if $(1 p>=1 / 3 /(\operatorname{gapd}(s) / 4-2 * \operatorname{gapc} 2(s) * \operatorname{gapdelta} 0(s)) * 4 *(\log (\log (3)+1 p)), 1,0)$

$\backslash \backslash$ Is $\log (p)$ larger than fourth minimal value on $\log p$ (base e)?

gapislogmin $4(s, I p)=$ if $(1 p>=1 / 3 /$ gapdelta0 $(s) *(4 * \log (\log (3)+1 p)-2 * \log (2) * \operatorname{gaplogc} 1(s)), 1,0)$

$\backslash \backslash$ Fifth minimal values on $\log p$, base 2

gaplogmin $(\mathrm{s})=\operatorname{gaplogc} 3(\mathrm{~s}) / \min (\operatorname{gapd}(\mathrm{s}) / 4$, gapdelta1 (s) /2)

$\backslash \backslash$ Constant $\mathrm{c}$ used in sixth minimal value $\operatorname{gapc}(\mathrm{s})=\operatorname{gaptau}(\mathrm{s}) / 96$

$\backslash \backslash$ Sixth minimal value on $\log p$, base 2

gaplogmin6 (s) $=\max (\log (17) / \log (2), 1+(2 / \operatorname{gaptau}(\mathrm{s}) / \operatorname{gapc}(\mathrm{s})) / \log (2))$

$\backslash \backslash$ Is $\log (\mathrm{p})$ larger than seventh minimal value on $\log \mathrm{p}$, base e gapislogmin $(s, l p)=$ if $(3 * 1 p * \operatorname{gapgamma}(s)>=\log (\operatorname{lp}-\log (2)), 1,0)$

$\backslash \backslash$ Eighth minimal value on $\log \mathrm{p}$, base 2

gaplogmin8 (s)=log (120)/ log (2)/3/gapgamma (s)

\\Minimum of $\log (p)$, base 2, for gapislogmin3

gapfind $3(s)=\{$

local $(j=2, i, k)$;

while(!gapislogmin $3(s, j), j=2 * j)$;

$\mathrm{k}=\mathrm{j} / 2$;

$i=\operatorname{ceil}((j+k) / 2)$;

while $(i !=j$,

if (!gapislogmin3 (s,i),

$k=i ; i=\operatorname{ceil}((j+k) / 2)$,

$j=i ; i=\operatorname{ceil}((j+k) / 2)))$;

ceil $(i / \log (2))$;

\}

$1 \backslash$ Minimum of $\log (p)$, base 2, for gapislogmin4 gapfind $4(\mathrm{~s})=\{$

local $(j=2, i, k)$;

while( ! gapislogmin $4(s, j), j=2 * j)$;

$\mathrm{k}=\mathrm{j} / 2$;

$i=\operatorname{ceil}((j+k) / 2)$;

while $(i !=j$,

if (! gapislogmin4 ( $\mathrm{s}, \mathrm{i})$,

$k=i ; i=\operatorname{ceil}((j+k) / 2)$,

$j=i ; i=\operatorname{ceil}((j+k) / 2)))$;

ceil (i/log(2));

\} 


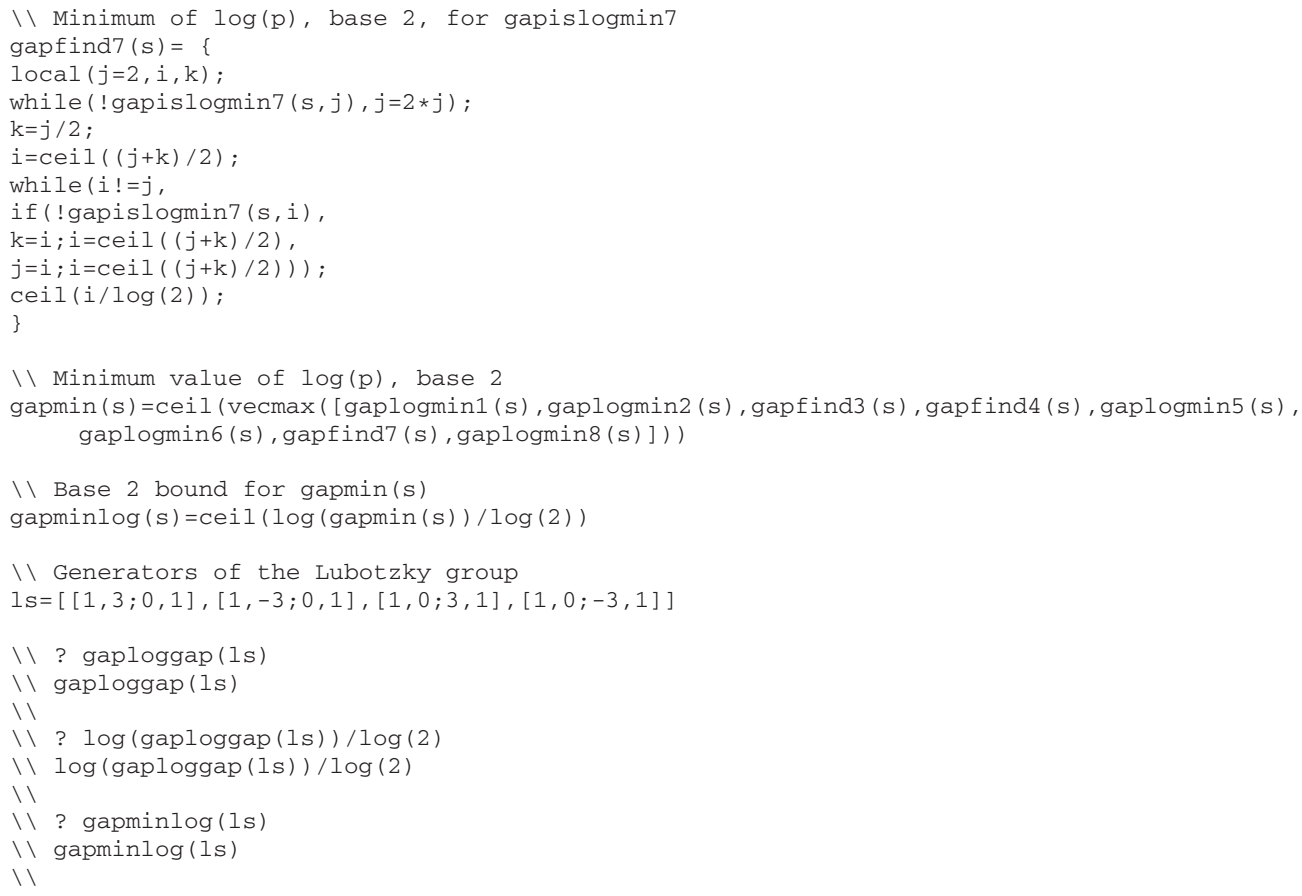

\section{Acknowledgement}

Much of the work on this paper was done during and following a course on expander graphs that I taught at ETH Zürich during the Fall Semester 2011. (Lecture notes for this course are available, and contain more background and motivating material [16].) Thanks to all those who attended this course and helped with corrections and remarks. Thanks in particular to O. Dinai, and to R. Pink for very interesting discussions and for helping with the proof of the specific variant of a "Larsen-Pink" inequality in Theorem 3.11. Thanks are also due to P. Sarnak for his comments, and especially for his insights concerning the history of questions and results concerning spectral gaps for subgroups of $\mathrm{SL}_{2}(\mathbf{Z})$. Thanks to L. Pyber for clarifying some of the "combinatorics" in the proof of the growth theorem.

Finally, I owe a great debt to the referee for his/her extremely detailed reading of the paper, which found many computational mistakes in the first draft. In a paper of this kind, this, of course, makes an enormous difference.

\section{A Appendix: proof of Theorem 2.1}

In this appendix, we sketch the proof of Theorem 2.1, following essentially line-by-line Tao's paper [24]. The presentation is therefore highly condensed, though we use a "diagram" notation which should make it relatively easy to check how the values of the constants evolve. 
Below all sets are subsets of a fixed finite group $G$, and are all nonempty.

\section{A.1 Diagrams}

We will use the following diagrammatic notation:

(1) If $A$ and $B$ are sets with Ruzsa distance

$$
d(A, B)=\log \left(\frac{\left|A \cdot B^{-1}\right|}{\sqrt{|A||B|}}\right)
$$

such that $d(A, B) \leq \log \alpha$, we write

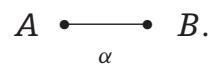

(2) If $A$ and $B$ are sets with $|B| \leq \alpha|A|$, we write

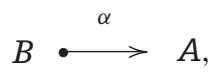

and, in particular, if $|X| \leq \alpha$, we write $X \stackrel{\alpha}{\longrightarrow}^{\prime} 1$,

(3) If $A$ and $B$ are sets with $e(A, B) \geq 1 / \alpha$, we write

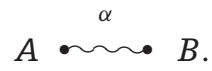

(4) If $A \subset B$, we also write $A>B$.

The following rules are easy to check (in addition to some more obvious ones which we do not spell out):

(1) From

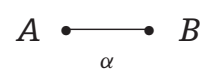

we can obtain

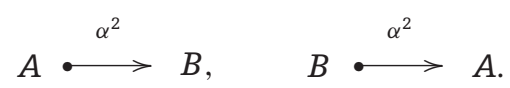


(2) (Ruzsa's triangle inequality) From

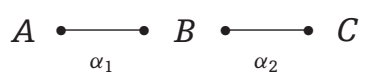

we obtain

$$
A \underset{\alpha_{1} \alpha_{2}}{\longrightarrow} \cdot C .
$$

(3) From

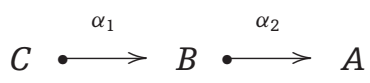

we obtain

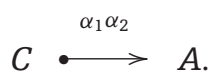

(4) (“Unfolding edges") From

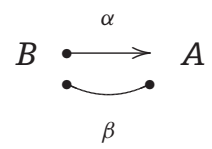

we obtain

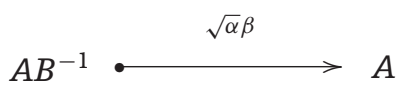

(note that by the first point in this list, we only need to have

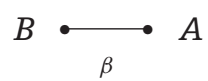

to obtain the full statement with $\alpha=\beta^{2}$, which is usually qualitatively equivalent.)

(5) ("Folding") From

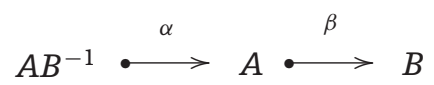

we obtain

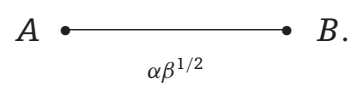


Note that the relation $A \stackrel{\alpha}{\longrightarrow} B$ is purely a matter of the size of $A$ and $B$, while the other arrow types depend on structural relations involving the sets (for $A>B$ ) and product sets (for $A \cdot \underset{\alpha}{\longrightarrow} B$ or $A \stackrel{\alpha}{\sim} B$ ).

\section{A.2 Proofs}

First, we state the Ruzsa covering lemma [24, Lemma 3.6] in our language:

Theorem A.1 (Ruzsa). If

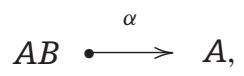

there exists a set $X$ which satisfies

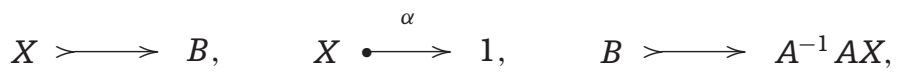

and symmetrically, if

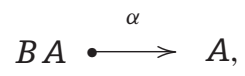

there exists $Y$ with

$$
Y>B, \quad Y \cdot \stackrel{\alpha}{\longrightarrow} 1, \quad B>Y A A^{-1} .
$$

Next, we have the link between sets with small tripling and approximate subgroups [24, Theorem 3.9 and Corollary 3.10]:

Theorem A.2. Let $A=A^{-1}$ with $1 \in A$ and

$$
A^{(3)} \stackrel{\alpha}{\longrightarrow} A
$$

Then $H=A^{(3)}$ is a $\left(2 \alpha^{5}\right)$-approximate subgroup containing $A$. 
Proof. We have first

$$
H \cdot \stackrel{\alpha}{\longrightarrow} A, \quad A>\longrightarrow H .
$$

Then, by Ruzsa's lemma 3.1, we obtain

$$
A H^{(2)}=A^{(7)} \stackrel{\alpha^{5}}{\longrightarrow} A,
$$

and by the Ruzsa covering lemma there exists $X$ with

$$
X>H^{(2)}, \quad X \cdot \stackrel{\alpha^{5}}{\longrightarrow} 1,
$$

such that

$$
H^{(2)} \longrightarrow A^{(2)} X \longrightarrow A^{(3)} X=H X
$$

Taking $X_{1}=X \cup X^{-1}$, we obtain a symmetric set with

$$
X_{1} \longrightarrow H^{(2)}, \quad X_{1} \stackrel{2 \alpha^{5}}{\longrightarrow} 1,
$$

and

$$
H^{(2)}>\quad \longrightarrow H X, \quad H^{(2)}>\longrightarrow X H,
$$

which are the properties defining a $\left(2 \alpha^{5}\right)$-approximate subgroup.

The next result is the explicit form of [24, Theorem 4.6, (i) implies (ii)]:

Theorem A.3. Let $A$ and $B$ with

$$
A \cdot \underset{\alpha}{\bullet} B^{-1}
$$

Then there exists a $\gamma$-approximate subgroup $H$ and a set $X$ with

$$
X \stackrel{\gamma}{1}^{\longrightarrow} 1, \quad A>X H, \quad B>H X, \quad H \cdot \stackrel{\gamma_{2}}{\longrightarrow} A,
$$


where

$$
\gamma \leq 2^{21} \alpha^{80}, \quad \gamma_{1} \leq 2^{28} \alpha^{108}, \quad \gamma_{2} \leq 8 \alpha^{14}
$$

Furthermore, one can ensure that

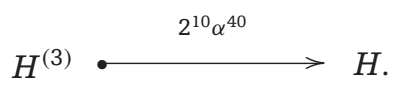

Proof. From

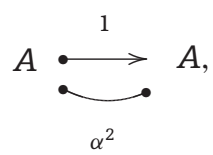

we first obtain

$$
A A^{-1} \stackrel{\alpha^{2}}{\longrightarrow} A \text {. }
$$

By [24, Proposition 4.5], we find a set $S$ with $1 \in S$ and $S=S^{-1}$ (the property $1 \in S$ is not explicitly stated in [24], but follows from the explicit definition used by Tao, namely $\left.S=\left\{x \in G|| A \cap A x\left|>\left(2 \alpha^{2}\right)^{-1}\right| A \mid\right\}\right)$ such that

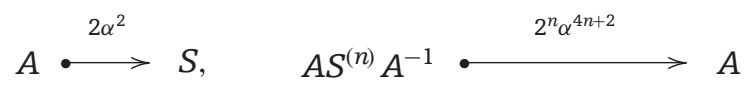

for all $n \geq 1$. In particular, we obtain

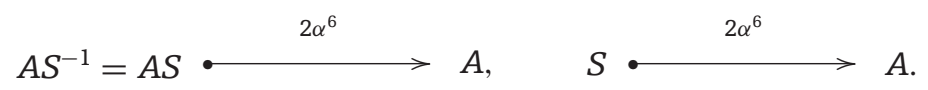

We have

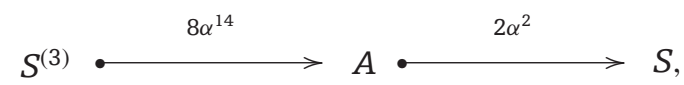

and Theorem A.2 says that $H=S^{(3)}$ is a $\gamma$-approximate subgroup containing $S$, with $\gamma=2\left(16 \alpha^{16}\right)^{5}=2^{21} \alpha^{80}$, and (as we see)

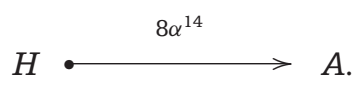


Moreover, we have

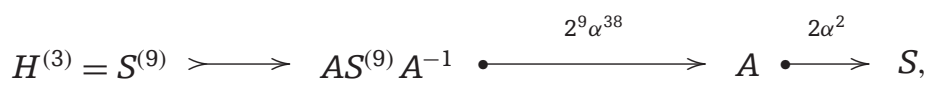

which gives (A.1).

Now from

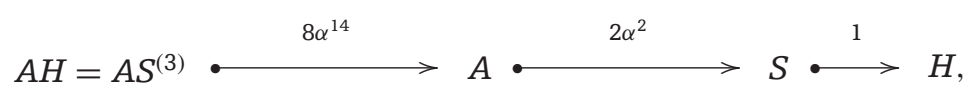

we see by the Ruzsa covering lemma that there exists $Y$ with

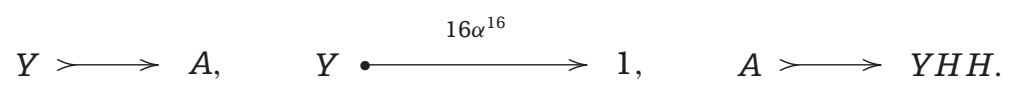

By definition of an approximate subgroup, there exists $Z$ with

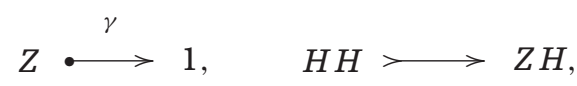

and hence

$$
A>(Y Z) H
$$

Now we go towards $B$. First, we have

$$
A H^{-1}=A S^{(3)} \bullet{ }^{8 \alpha^{14}} \longrightarrow A \cdot \stackrel{2 \alpha^{2}}{\longrightarrow} H
$$

which, again by folding, gives

$$
A \cdot \underset{\alpha_{1}}{\longrightarrow} H
$$

with $\alpha_{1}=8 \sqrt{2} \alpha^{15}$. Hence, we can write

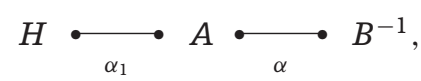


and so

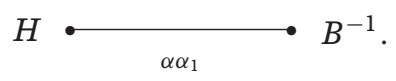

In addition, we have

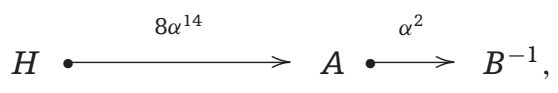

and therefore we obtain

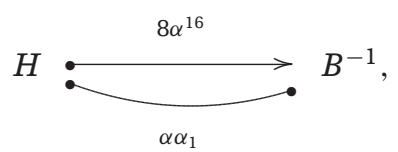

from which it follows by unfolding that

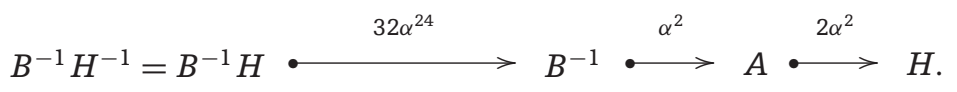

Once more by the Ruzsa covering lemma, we find $Y_{1}$ with

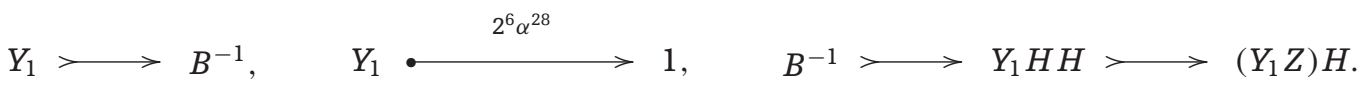

Now, we need only take $X=\left(Y_{1} Z \cup Y Z\right)$, so that

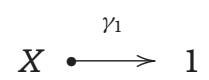

with $\gamma_{1}=\gamma\left(64 \alpha^{28}+16 \alpha^{16}\right)$, in order to conclude. Since

$$
\gamma_{1} \leq 2^{28} \alpha^{108}
$$

we are done.

The next result is a version of the Balog-Gowers-Szemerédi Lemma found in [24, Theorem 5.2]. 
Theorem A.4. Let $A$ and $B$ with

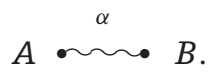

Then there exist $A_{1}$ and $B_{1}$ with

$$
A_{1} \longrightarrow A, \quad B_{1} \longrightarrow B
$$

as well as

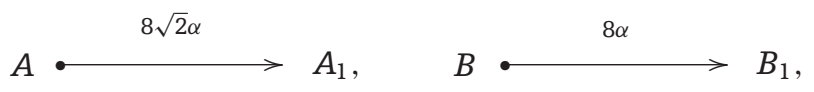

and

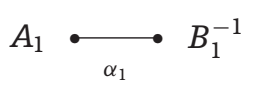

where $\alpha_{1}=2^{23} \alpha^{9}$.

This is not entirely spellt out in [24], but only the last two or three inequalities in the proof need to be made explicit to obtain this value of $\alpha_{1}$. Finally, the next theorem is just the "diagrammatic" version of Theorem 2.1, and therefore completes its proof. It is an explicit version of [24, Theorem 5.4; (i) implies (iv)].

Theorem A.5. Let $A$ and $B$ with

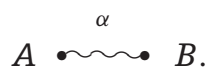

Then there exist a $\beta$-approximate subgroup $H$ and $x, y \in G$, such that

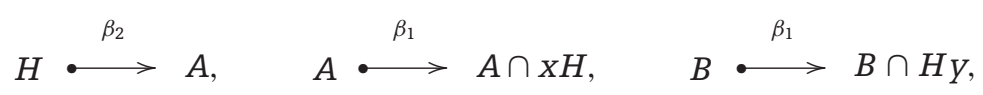

where

$$
\beta \leq 2^{1861} \alpha^{720}, \quad \beta_{1} \leq 2^{2516} \alpha^{973}, \quad \beta_{2} \leq 2^{325} \alpha^{126} .
$$


Moreover, one can ensure that

$$
H^{(3)} \stackrel{\beta_{3}}{\longrightarrow} H
$$

where $\beta_{3}=2^{930} \alpha^{360}$.

Proof. By the Balog-Gowers-Szemerédi Theorem, we obtain $A_{1}$ and $B_{1}$ with

$$
A_{1} \longrightarrow A, \quad B_{1} \longrightarrow B
$$

as well as

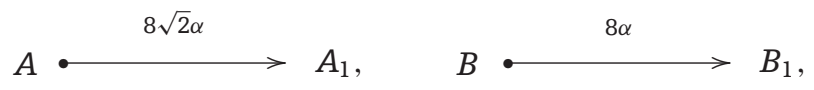

and

$$
A_{1} \cdot \underset{\alpha_{1}}{\bullet} B_{1}^{-1}
$$

where $\alpha_{1}=2^{23} \alpha^{9}$. Applying Theorem A.3 to $A_{1}$ and $B_{1}$, we obtain a $\beta$-approximate subgroup $H$ and a set $X$ with

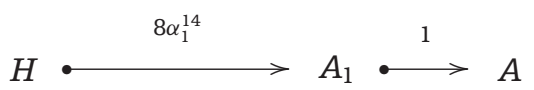

and

$$
X \stackrel{\gamma}{\longrightarrow} 1, \quad A_{1} \longrightarrow X H, \quad B_{1} \longrightarrow H X,
$$

where

$$
\beta=2^{21} \alpha_{1}^{80}=2^{1861} \alpha^{720}, \quad \gamma=2^{28} \alpha_{1}^{108}=2^{2512} \alpha^{972},
$$

and moreover

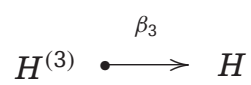

where $\beta_{3}=2^{10} \alpha_{1}^{40}=2^{930} \alpha^{360}$. 
Applying the pigeonhole principle, we find $x$ such that

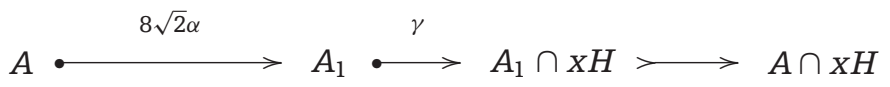

and $y$ with

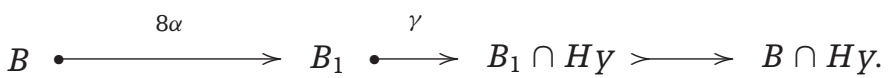

This gives what we want with

$$
\beta_{1} \leq 8 \sqrt{2} \alpha \gamma \leq 2^{2516} \alpha^{973}, \quad \beta_{2}=8 \alpha_{1}^{14}=2^{325} \alpha^{126} .
$$

\section{References}

[1] Bourgain, J. and A. Gamburd. "Uniform expansion bounds for Cayley graphs of $\mathrm{SL}_{2}\left(\mathbf{F}_{p}\right)$." Annals of Mathematics 167, no. 2 (2008): 625-42.

[2] Bourgain, J., A. Gamburd, and P. Sarnak. "The affine linear sieve." Inventiones mathematicae 179, no. 3 (2010): 559-644.

[3] Breuillard, E. and T. Gelander. "Uniform independence in linear groups." Inventiones mathematicae 173, no. 2 (2008): 225-63.

[4] Breuillard, E., B. Green, and T. Tao. "Approximate subgroups of linear groups." Geometric and Functional Analysis 21, no. 4 (2011): 774-819; arXiv:1005.1881.

[5] Carter, R. W. Finite groups of Lie Type. New York, NY: Wiley Interscience 1985.

[6] Digne, F. and J. Michel. Representations of Finite Groups of Lie Type, London Mathematical Society Student Texts 21. Cambridge, UK: Cambridge University Press, 1991.

[7] Fulton, W. and J. Harris. Representation Theory. Graduate Texts in Mathematics 129. Berlin: Springer, 1991.

[8] Gamburd, A. "On the spectral gap for infinite index "congruence" subgroups of $\mathrm{SL}_{2}$ (Z)." Israel Journal of Mathematics 127 (2002): 157-200.

[9] Glibichuk, A. A. and S. V. Konyagin. "Additive Properties of Product Sets in Fields of Prime Order." In Additive Combinatorics, 279-286. C.R.M. Proceedings and Lecture Notes 43. Providence, RI: American Mathematical Society, 2006.

[10] Gowers, W. T. "Quasirandom groups." Combinatorics, Probability and Computer Science 17, no. 3 (2008): 363-87.

[11] Helfgott, H. "Growth and generation in $\mathrm{SL}_{2}(\mathbf{Z} / p \mathbf{Z})$." Annals of Mathematics 167, no. 2 (2008): 601-23.

[12] Hrushovski, E. "Stable group theory and approximate subgroups." Journal of the American Mathematical Society 25, no. 1 (2012): 189-243. 
[13] Huxley, M. "Exceptional eigenvalues and congruence subgroups." In The Selberg Trace Formula and Related Topics, edited by D. Hejhal, P. Sarnak and A. Terras, 341-9. Contemporary Mathematics 53. Providence, RI: American Mathematical Society, 1986.

[14] Kesten, H. "Symmetric random walks on groups." Transactions of the American Mathematical Society 92 (1959): 336-54.

[15] Kowalski, E. Sieve in Expansion, Séminaire, exposé 1028 (2010); Astérisque, to appear.

[16] Kowalski, E. Expander Graphs. Lecture notes for ETH Zürich Fall Semester course; www.math.ethz.ch/ kowalski/expander-graphs.pdf

[17] Larsen, M. and R. Pink. "Finite subgroups of algebraic groups." Journal of the American Mathematical Society 24 (2011): 1105-58.

[18] Levin, D., Y. Peres, and E. Wilmer. Markov Chains and Mixing Times. Providence, RI: American Mathematical Society, 2009.

[19] Nikolov, N. and L. Pyber. "Product decompositions of quasirandom groups and a Jordantype theorem." Journal of European Mathematical Society 13 (2011): 1063-77.

[20] PARI/GP, version 2.6.0, Bordeaux, 2011, http://pari.math.u-bordeaux.fr/.

[21] Pyber, L. and E. Szabó. "Growth in finite simple groups of Lie type of bounded rank." (2010): preprint arXiv:1005.1858v1.

[22] Sarnak, P. and X. Xue. "Bounds for multiplicities of automorphic representations." Duke Mathematical Journal 64 (1991): 207-27.

[23] Suzuki, M. Group Theory, I, Grundlehren der Mathematischen Wissenschaften 247. Berlin: Springer, 1982.

[24] Tao, T. "Product set estimates for non-commutative groups." Combinatorica 28 (2008): 547-94.

[25] Tao, T. "An elementary non-commutative Freiman theorem." terrytao.wordpress.com/ 2009/11/10/an-elementary-non-commutative-freiman-theorem.

[26] Tao, T. and V. Vu. Additive Combinatorics, Cambridge Studies Advanced Mathematics 105. Cambridge, UK: Cambridge University Press, 2006. 